\title{
Genomic and transcriptomic analysis of carbohydrate utilization by Paenibacillus sp. JDR-2: systems for bioprocessing plant polysaccharides
}

Neha Sawhney ${ }^{1 \dagger}$, Casey Crooks ${ }^{2}$, Virginia Chow ${ }^{1}$, James F. Preston ${ }^{1 *}$ and Franz J. St John ${ }^{2^{*+}}$

\begin{abstract}
Background: Polysaccharides comprising plant biomass are potential resources for conversion to fuels and chemicals. These polysaccharides include xylans derived from the hemicellulose of hardwoods and grasses, soluble $\beta$-glucans from cereals and starch as the primary form of energy storage in plants. Paenibacillus sp. JDR-2 (Pjdr2) has evolved a system for bioprocessing xylans. The central component of this xylan utilization system is a multimodular glycoside hydrolase family 10 ( $\mathrm{GH} 10)$ endoxylanase with carbohydrate binding modules (CBM) for binding xylans and surface layer homology (SLH) domains for cell surface anchoring. These attributes allow efficient utilization of xylans by generating oligosaccharides proximal to the cell surface for rapid assimilation. Coordinate expression of genes in response to growth on xylans has identified regulons contributing to depolymerization, importation of oligosaccharides and intracellular processing to generate xylose as well as arabinose and methylglucuronate. The genome of Pjdr2 encodes several other putative surface anchored multimodular enzymes including those for utilization of $\beta-1,3 / 1,4$ mixed linkage soluble glucan and starch.

Results: To further define polysaccharide utilization systems in Pjdr2, its transcriptome has been determined by RNA sequencing following growth on barley-derived soluble $\beta$-glucan, starch, cellobiose, maltose, glucose, xylose and arabinose. The putative function of genes encoding transcriptional regulators, $A B C$ transporters, and glycoside hydrolases belonging to the corresponding substrate responsive regulon were deduced by their coordinate expression and locations in the genome. These results are compared to observations from the previously defined xylan utilization systems in Pjdr2. The findings from this study show that Pjdr2 efficiently utilizes these glucans in a manner similar to xylans. From transcriptomic and genomic analyses we infer a common strategy evolved by Pjdr2 for efficient bioprocessing of polysaccharides.

Conclusions: The barley $\beta$-glucan and starch utilization systems in Pjdr2 include extracellular glycoside hydrolases bearing CBM and SLH domains for depolymerization of these polysaccharides. Overlapping regulation observed during growth on these polysaccharides suggests they are preferentially utilized in the order of starch before xylan before barley $\beta$-glucan. These systems defined in Pjdr2 may serve as a paradigm for developing biocatalysts for efficient bioprocessing of plant biomass to targeted biofuels and chemicals.
\end{abstract}

Keywords: Paenibacillus sp. JDR-2, Xylans, Soluble $\beta$-glucan, Starch, Bioprocessing systems, Transcriptome, RNA sequencing, Biofuels and chemicals

\footnotetext{
*Correspondence: jpreston@ufl.edu; fjstjohn@gmail.com

${ }^{\dagger}$ Equal contributors

'Department of Microbiology and Cell Science, University of Florida,

Gainesville, FL 32611, USA

${ }^{2}$ Institute for Microbial and Biochemical Technology, Forest Products

Laboratory, USDA Forest Service, Madison, WI 53726, USA
} 


\section{Background}

The bacterium Paenibacillus sp. JDR-2 (Pjdr2) originally isolated from sweetgum wood (Liquidambar styraciflua) disks exposed to surface soils has been shown to completely utilize the lignocellulosic polymer glucuronoxylan $\left(\mathrm{GX}_{\mathrm{n}}\right)$. Previous studies showed that growth on minimal media supplemented with polymeric xylan was preferred to that on simple sugars such as xylose, glucose, or arabinose $[1,2]$. These studies indicated that efficient xylan utilization is attributable, in part, to a $157 \mathrm{kDa}$ GH10 $\beta$-1,4-endoxylanase (Xyn10A ${ }_{1}$ ) containing carbohydrate binding modules (CBM) for binding to polysaccharides and surface layer homology (SLH) domains for cell-association. The efficiency of utilization was such that the products of xylan hydrolysis were rapidly assimilated as they were formed $[2,3]$. These early findings suggest that Pjdr2 utilizes glucuronoxylan in a vectorial manner with an unidentified mechanism for coupling surface localized polymer hydrolysis to rapid oligoxyloside transport into the cell.

PCR screening for genes encoding enzymes typically involved in utilization of $\mathrm{GX}_{\mathrm{n}}$ led to the identification of the aldouronate utilization gene cluster (Fig. 1a). This cluster of genes encodes three intracellular glycoside hydrolases, a GH67 $\alpha$-glucuronidase, a GH10 endoxylanase and a GH43

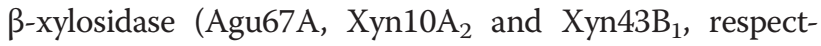
ively), as well as regulatory proteins and $\mathrm{ABC}$ (ATP-binding cassette) transporters (Fig. 1a and Additional file 1).
Through in silico analysis this gene cluster is predicted to contain multiple promoters and catabolite repression elements (cre) although the entire region has only a single detected terminator following the last gene, $x y n 43 B_{1}$. Importantly, the aldouronate utilization gene cluster was coordinately regulated with the distally located $x y n 10 A_{1}$, supporting the role of this large surface anchored xylanase in membrane localized glucuronoxylan hydrolysis [4]. More recent studies showed that Pjdr2 utilized polymeric $\mathrm{GX}_{\mathrm{n}}$ at a rate 2.8 times higher than the $\mathrm{GX}_{\mathrm{n}}$ derived aldouronate, aldotetrauronic acid, suggesting a functional coupling of primary xylan hydrolysis to oligosaccharide transport into the cell [3].

Analysis of the sequenced genome [5] for carbohydrate active enzymes (CAZy) through the CAZy database (http://www.cazy.org/) [6] identified a number of genes encoding enzymes predicted to be involved in xylan utilization. These include five genes encoding GH10 and one encoding GH11 xylanases with expected, and in three cases demonstrated, endo- $\beta-1,4-x y l a n a s e$ activity for xylan main chain hydrolysis. Genes encoding accessory enzymes for xylan depolymerization including $\beta$-xylosidases, $\alpha$ glucuronidases, $\alpha$-L-arabinofuranosidases, and acetyl esterases are also predicted to be numerous. For example, the genome of Pjdr2 contains 25 genes encoding a broad class of GH43 enzymes including $\beta$-xylosidases and $\alpha$ $\mathrm{L}$-arabinofuranosidases. There are also four genes encoding $\alpha$-glucuronidases of which three are in the

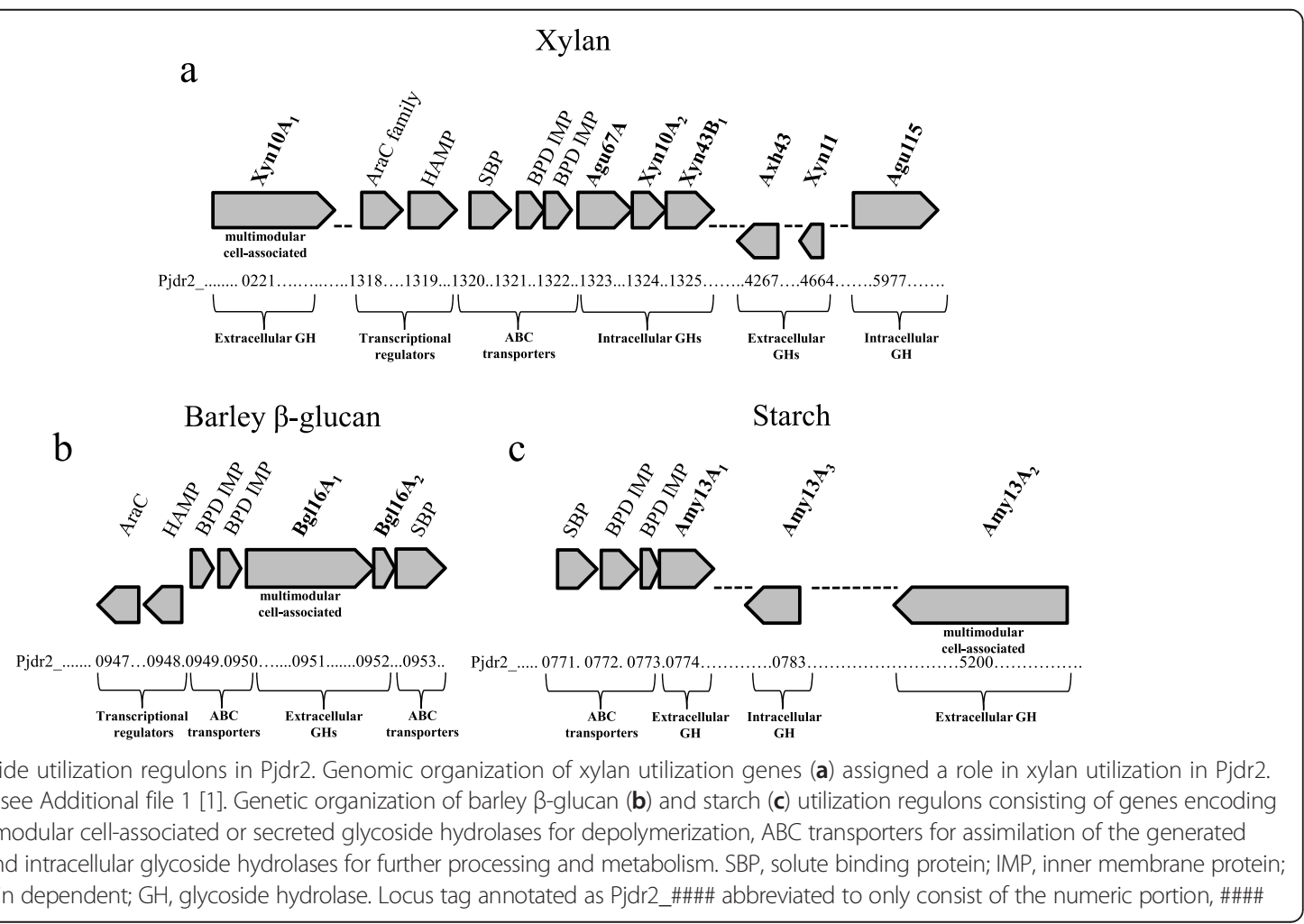


GH115 family and one in the GH67 family. Recombinant enzymes encoded by several of these genes have been characterized $[2,3,7]$. Several other CAZy families commonly involved in xylan utilization are also highly represented. The cumulative potential xylan degrading capacity of Pjdr2 supports its demonstrated abilities for complete xylan utilization.

More recent efforts have focused on transcriptomic analysis of Pjdr2 while growing on xylan substrates. Proteins involved in xylan utilization were identified based on gene expression levels [1]. Primary findings of that study revealed that Pjdr2 secretes only two endoxylanases for xylan depolymerization. The resulting mixture of oligosaccharides generated by Xyn10A $\mathrm{A}_{1}$ [2] and Xyn11 (unpublished) consists of neutral oligoxylosides and, depending on the xylan type, a mixture of substituted xylooligosaccharides including aldouronates and similarly substituted oligoarabinoxylosides. Genes predicted to encode $A B C$ transporters were found to have increased transcript levels during growth on xylans and it was proposed that these enable transport of this complex mixture of oligoxylosides into the cell for intracellular hydrolysis to monomeric sugars for catabolism $[2-4,8]$. The observed high expression level of genes encoding intracellular oligosaccharide degrading accessory enzymes during growth on xylans supported this hypothesis (Additional file 1). Comparative transcriptomics following growth of Pjdr2 on dicot-derived hardwood (sweetgum) $\mathrm{GX}_{\mathrm{n}}$ and a monocot-derived grass (sorghum) glucuronoarabinoxylan $\left(\mathrm{GAX}_{\mathrm{n}}\right)$ indicated that systems with distinct enzymes and $\mathrm{ABC}$ transporters are employed for utilization of oligoarabinoxylosides as compared to aldouronates [1].

Based on the earlier physiological characterization and the most recent transcriptomic study, the proposed model for xylan utilization indicates that Pjdr2 relies on transport and intracellular degradation of xylooligosaccharides. This system in Pjdr2 shows similarity to the polymeric sugar utilization by other bacteria including Geobacillus, Thermotoga and Clostridium [9-11] and stands in contrast to the more classical paradigm for fungi that requires complete extracellular conversion of polysaccharides to monosaccharides [12]. The role of the cell-associated endoxylanase in the xylan utilization systems represents an alternative paradigm to that observed in cellulolytic bacteria such as Clostridium thermocellum in which glycoside hydrolases comprise a cell-associated complex as opposed to individual enzymes [13-16].

Recent studies show that Pjdr2 is also capable of efficient utilization of other biomass derived polysaccharides including barley $\beta$-glucan and starch $[17,18]$. Genome analysis indicates that these polysaccharide utilization systems include extracellular glycoside hydrolases with modular architecture for cell-association and carbohydrate binding. We present here an overview of a broad transcriptomic study characterizing Pjdr2 gene regulation in response to growth on barley $\beta$-glucan and starch as well as their constituent disaccharide sugars, cellobiose and maltose. The results are additionally considered in regards to the previously studied xylanutilization system, providing a comparison of these three polysaccharide-utilization systems with respect to transport and catabolism of the products of depolymerization as well as their monosaccharide constituents. Comparison of these three polysaccharide utilization systems of Pjdr2 indicate a reliance upon cell-associated glycoside hydrolases with CBM's for interacting with polysaccharides and SLH domains for cell-association. Furthermore, identification of 29 genes within the Pjdr2 genome encoding proteins involved in carbohydrate utilization that contain sets of SLH domains supports an evolutionary path leading to the secretion of cellassociated glycoside hydrolases. This system is efficient in the depolymerization of polysaccharides at the cell surface and is found in Pjdr2 as well as related bacteria including Clostridium [19-21], Caldicellulosiruptor [22] and Thermoanaerobacter [23].

\section{Results and discussion \\ Experimental design}

For this transcriptome study, we sought a greater understanding of how Pjdr2 utilizes polymeric sugars. Genome analysis and polysaccharide growth studies supported efficient utilization of the polysaccharides soluble $\beta$-glucan and starch. Through bioconversion these abundant biomass-derived sugar polymers may contribute to the production of value-added chemical or fuels. To obtain a broad understanding of how these polysaccharides are utilized by Pjdr2, total RNA was prepared from earlymid exponentially growing cultures growing on these polysaccharides as well as their limit enzymatic hydrolysis products and their constituent simple sugars. The sample preparation and RNA-seq data acquisition portions of this work overlap with a recently published $x y-$ lan utilization transcriptome and the results presented here are compared with this earlier work to provide perspective, draw conclusions and identify themes which define the efficient manner in which Pjdr2 utilizes polysaccharides [1]. The saccharides used in the study that provided the final comparative data set include barley $\beta$ glucan (B) and cellobiose (C) representing $\beta$-configured glucans, starch $(\mathrm{S})$ and maltose $(\mathrm{M})$ representing $\alpha$ configured glucans, sweetgum glucuronoxylan (SG) and sorghum glucuronoarabinoxylan (SO) representing different xylan types and the constituent monosaccharides of these polysaccharides, including glucose $(\mathrm{G})$, xylose (X) and arabinose (A). Further, each condition was routinely compared to a yeast extract (YE) control 
condition which consisted of $0.5 \%$ YE without added carbohydrate and a sweetgum xylan with no YE (SGnoYE) control. Throughout the manuscript where specific genes are considered, their respective transcript levels or their encoded proteins are routinely identified with an accompanying abbreviated locus tag accession number consisting of the four digit number, e. g. the locus tag Pjdr2_0001 would be described as 0001. The total data set normalized RPKM (Reads Per Kilobase per Million reads sequenced) values were compared by fold changes taken from the ratio of the condition in consideration over the YE control condition unless otherwise stated. Data was judged to be significant given a 4-fold change and a $p$-value, $<0.05$.

\section{Genes involved in barley $\beta$-glucan utilization}

Recent studies have shown that Pjdr2 may utilize soluble $\beta$-glucans [18]. Barley $\beta$-glucan consists of a linear polysaccharide chain of $\beta-1,4$ linked glucose frequently and regularly interrupted with $\beta$-1,3-linked glucose [24]. This polysaccharide lacks side chain substitutions such as those found in xylan, hence the extracellular degradation of barley- $\beta$-glucan is correspondingly less complex, presumably requiring fewer enzymes. The genome of Pjdr2 encodes three GH16 enzymes (genes 0951, 0952, and 0824) annotated as licheninases or laminarinases through domain analysis. All three enzymes are predicted to be secreted and while one consists of a singular GH16 catalytic module, the other two have an extensive multimodular architecture. Both of these modular enzymes (genes 0951 and 0824) contain triplicate N-terminal SLH domains, presumably for cell surface localization, and multiple CBM's similar to those observed for the Xyn10A 1 enzyme involved in xylan utilization (Fig. 4).

During growth on barley- $\beta$-glucan, the genes encoding the multimodular $\mathrm{Bgl16A}_{1}$ (gene 0951) and the non-modular Bgl16 $\mathrm{A}_{2}$ (gene 0952) increased 80-fold and 25 -fold respectively compared to growth on the yeast extract control (YE) not supplemented with carbohydrate (Table 1). The $b g l 16 A_{3}$ gene (0824) encoding the second large multimodular GH16 enzyme is expressed only at low levels on all substrates tested. Bgl16 $\mathrm{A}_{1}$ shares $34 \%$ amino acid similarity with the catalytic domain of the GH16 laminarinase from Thermotoga maritima (UniProt accession: Q9WXN1) [25] and Bgl16A $\mathrm{A}_{2}$ shares $71 \%$ similarity to a probable licheninase from Bacillus subtilis (UniProt accession: P04957) (Table 2) [26]. In support of these annotations, recombinant $\mathrm{Bgl}_{16 \mathrm{~A}_{1}}$ has been shown to have activity against barley $\beta$-glucan and laminarin as both substrates contain the requisite $\beta$ 1,3 -glucan linkage while recombinant $\mathrm{Bgl}_{16 \mathrm{~A}_{2}}$ shows its highest activity on barley $\beta$-glucan [18].
Hydrolysis of barley $\beta$-glucan with GH16 laminarinase and licheninase enzymes is expected to liberate $\beta-1,3 / 1,4$ mixed linkage glucooligosaccharides. Increased transcript levels for two predicted $\mathrm{ABC}$ transporter gene cassettes were observed during growth on barley $\beta$-glucan compared to the YE control. The first cassette consisting of genes 0949, 0950 and 0953, flanks the enzyme encoding genes $b g l 16 A_{1}$ and $b g l 16 A_{2}$ described above and showed greater than a 1200 -fold increase in transcript levels during growth on barley $\beta$-glucan (Table 3 ). These barley $\beta$-glucan utilization genes constitute an apparent operon specifically responsive to growth on soluble $\beta-1,3$ (4)-glucans and no other tested substrate. This operon is directly linked to a $\beta$-glucan-responsive set of putative transcriptional regulators (genes 0947 and 0948) located immediately upstream but transcribed in the opposite direction. Together, these seven genes, 0947 through 0953, constitute the glucan utilization gene cluster (Fig. 1b). The second $\mathrm{ABC}$ transporter gene cassette consisting of genes 5314,5315 , and 5316 has increased expression on barley $\beta$-glucan and was also increased on xylan [1]. This overlapping regulation will be discussed below.

Comparison of the barley $\beta$-glucan utilization system described here to the xylan utilization system described previously [1] implies a missing enzymatic component for barley $\beta$-glucan utilization. Pjdr2 appears to transport the mixed linkage oligosaccharide products of the two secreted GH16 endoglucanases in a manner similar to xylan utilization. However, unlike the defined intracellular oligosaccharide processing in the xylan utilization systems, there is no clear evidence for increased expression of genes encoding enzymes for intracellular hydrolysis of glucooligosaccharides contributing to the barley $\beta$-glucan utilization system. Genome analysis has identified several genes encoding enzymes which could be involved in the further processing of intracellular glucooligosaccharides (e.g. the genome encodes fifteen GH3 enzymes), but none of these genes are confidently assigned to this role based on the transcriptomic data. One candidate, gene 0317, encoding an intracellular GH3 $\beta$-glucosidase attains elevated transcript levels with growth on barley- $\beta$-glucan and cellobiose. The comparatively high expression level on YE resulted in a limited relative increase of just 2.7 -fold with barley $\beta$-glucan, although compared to growth on arabinose or glucose this gene had a 10 and 5.4-fold increase in expression, respectively (Table 1).

\section{Genes with increased expression during growth on cellobiose}

Cellobiose is thought to represent a primary limit product of mixed linkage $\beta$-glucan utilization and was chosen for study to discriminate between utilization of the barley $\beta$-glucan polymer and its hydrolysis products. 
Table 1 Expression analysis of polysaccharide processing genes during growth on barley $\beta$-glucan, starch and maltose

\begin{tabular}{|c|c|c|c|c|c|c|c|c|c|c|c|c|c|c|c|}
\hline \multirow[t]{2}{*}{ Family $^{a}$} & \multirow[t]{2}{*}{$\mathrm{LT}^{\mathrm{b}}$} & \multirow[t]{2}{*}{ Protein product } & \multirow[t]{2}{*}{ Name $^{c}$} & \multirow[t]{2}{*}{$S P^{d}$} & \multicolumn{5}{|c|}{ Fold change } & \multicolumn{6}{|c|}{ Linear RPKM Values ${ }^{f}$} \\
\hline & & & & & $\mathrm{B} / \mathrm{YE}$ & $C / Y E$ & $\mathrm{G} / \mathrm{YE}$ & S/YE & $M / Y E$ & B & C & G & S & M & YE \\
\hline \multicolumn{16}{|c|}{ Barley $\beta$-glucan utilization } \\
\hline $\mathrm{GH} 16$ & 0951 & laminarinase & Bgl16 $A_{1}$ & Yes & $\underline{80.3}$ & 0.2 & 0.2 & 0.4 & 0.1 & 170.2 & 0.4 & 0.5 & 0.8 & 0.3 & 2.1 \\
\hline GH16 & 0952 & endo- $\beta-1,3-1,4$ glucanase & $\mathrm{Bgl} 16 \mathrm{~A}_{2}$ & Yes & $\underline{24.5}$ & 0.2 & 0.2 & 0.3 & 0.1 & 124.1 & 1.1 & 0.8 & 1.5 & 0.6 & 5.1 \\
\hline $\mathrm{GH} 16$ & 0824 & laminarinase & $\mathrm{Bgl} 16 \mathrm{~A}_{3}$ & Yes & 0.4 & 0.5 & 0.6 & 0.6 & 0.6 & 1.7 & 2.2 & 2.7 & 2.4 & 2.4 & 4.1 \\
\hline $\mathrm{GH}^{\mathrm{g}}$ & 0317 & glycoside hydrolase & & No & $\underline{2.7}$ & $\underline{2.7}$ & 0.5 & NS & $\underline{2.4}$ & 379.0 & 376.6 & 70.7 & 156.1 & 338.1 & 141.5 \\
\hline \multicolumn{16}{|c|}{ Barley $\beta$-glucan induced xylanases } \\
\hline GH67 & 1323 & a-glucuronidase & Agu67A & No & $\underline{20.6}$ & 0.8 & 0.4 & 0.6 & 0.4 & 120.2 & 4.4 & 2.1 & 3.5 & 2.5 & 5.8 \\
\hline $\mathrm{GH} 8$ & 1182 & exooligoxylanase & Xyn8 & No & $\underline{39.3}$ & 0.4 & 0.4 & 0.7 & 0.5 & 297.2 & 3.3 & 3.1 & 5.1 & 3.5 & 7.6 \\
\hline $\mathrm{GH} 11$ & 4664 & endoxylanase & Xyn11 & Yes & $\underline{3.7}$ & $0.4^{*}$ & 0.2 & $0.3^{*}$ & 0.2 & 7.5 & 0.8 & 0.4 & 0.5 & 0.4 & 2.1 \\
\hline $\mathrm{GH} 10$ & 0221 & endoxylanase & $\mathrm{Xyn}_{10 \mathrm{~A}_{1}}$ & Yes & $\underline{8.8}$ & 0.2 & 0.1 & 0.3 & 0.1 & 52.0 & 1.2 & 0.8 & 1.6 & 0.6 & 5.9 \\
\hline $\mathrm{GH} 10$ & 1324 & endoxylanase & Xyn $10 A_{2}$ & No & $\underline{24.0}$ & $0.8^{*}$ & 0.5 & $0.6^{*}$ & 0.6 & 185.3 & 6.2 & 3.5 & 4.7 & 4.4 & 7.7 \\
\hline $\mathrm{GH} 43$ & 1325 & xylosidase & $X_{1 y n} 43 B_{1}$ & No & $\underline{19.6}$ & $0.9^{*}$ & 0.5 & $0.7^{*}$ & $0.8^{*}$ & 222.1 & 9.9 & 5.9 & 7.6 & 8.6 & 11.3 \\
\hline $\mathrm{GH} 43$ & 0750 & xylosidase & Xyn43B 2 & No & $\underline{3.2}$ & 176.6 & NS & NS & NS & 5.0 & 276.7 & 2.7 & 2.6 & 1.8 & 1.6 \\
\hline $\mathrm{GH} 43$ & 1907 & xylosidase & Xyn43B 3 & No & $\underline{111.5}$ & $\underline{2.3}$ & NS & NS & 0.6 & 1410.9 & 29.6 & 12.2 & 13.5 & 7.6 & 12.7 \\
\hline \multicolumn{16}{|c|}{ Starch utilization } \\
\hline $\mathrm{GH} 13$ & 0774 & a-amylase & Amy $13 A_{1}$ & Yes & 0.2 & 0.2 & 0.1 & 114.1 & $\underline{69.7}$ & 2.0 & 1.8 & 1.3 & 1127.3 & 688.4 & 9.9 \\
\hline GH13 & 5200 & a-amylase & Amy $13 \mathrm{~A}_{2}$ & Yes & 0.2 & 0.2 & 0.2 & $\underline{56.8}$ & $\underline{4.2}$ & 1.5 & 1.5 & 1.2 & 395.7 & 29.3 & 7.0 \\
\hline $\mathrm{GH} 13$ & 0783 & a-amylase & Amy $13 \mathrm{~A}_{3}$ & No & 1.5 & NS & NS & 112.2 & $\underline{95.6}$ & 7.8 & 5.7 & 6.5 & 584.3 & 497.9 & 5.2 \\
\hline $\mathrm{GH} 13$ & 1045 & a-amylase & Amy $13 \mathrm{~A}_{4}$ & No & NS & 2.7 & 3.8 & NS & NS & 2.6 & 4.7 & 6.6 & 1.5 & 1.4 & 1.7 \\
\hline $\mathrm{GT}^{\mathrm{g}}$ & 1149 & a-glucan phosphorylase & MalP & No & 0.3 & 0.1 & 0.5 & NS & 0.2 & 2.4 & 0.9 & 0.7 & 6.6 & 1.5 & 8.9 \\
\hline \multicolumn{16}{|c|}{ Maltose utilization } \\
\hline ND & 5587 & oxidoreductase & ThuB & No & NS & NS & NS & NS & $\underline{8.8}$ & 7.4 & 7.0 & 7.3 & 9.0 & 73.8 & 8.4 \\
\hline GATase1 & 5588 & hypothetical protein & ThuA & No & 0.7 & 0.7 & NS & NS & $\underline{8.1}$ & 7.1 & 2.6 & 2.5 & 2.5 & 30.3 & 3.8 \\
\hline
\end{tabular}

${ }^{\mathrm{a}} \mathrm{GH}$, glycoside hydrolase; GT, glycosyltransferase; ND, not determined; GATase1, type 1 glutamine amidotransferase (GATase1)-like domain

${ }^{\mathrm{b}} \mathrm{LT}$, locus tag annotated as Pjdr2_\#\#\#\# abbreviated to only consist of the numeric portion, \#\#\#\#

'The name assigned to gene candidates with enzymes characterized in our laboratory in bold

${ }^{\mathrm{d}} \mathrm{SP}$, sequence encodes a predicted signal peptide for secretion

${ }^{\mathrm{e}}$ Transcript levels of candidate genes that were expressed 2-fold greater (underlined) and those that were expressed 4-fold greater (bold) than the yeast extract without carbohydrate growth are indicated. The growth substrates are shown as follows: B, barley $\beta$-glucan; $C$, cellobiose; G, glucose; $S$, starch; $M$, maltose; YE, yeast extract. Significance of fold change data is judged by having a $p$-value no more than 0.01 . Data with $p$-values between 0.01 and 0.05 are denoted with an asterisk, and those with $p$-values greater than 0.05 are designated as not significant (NS)

fRPKM values are defined as Reads Per Kilobase per Million reads sequenced

${ }^{9}$ Gene 0317 and gene 1149 are included in this table as genes of interest in barley $\beta$-glucan and starch utilization pathways, respectively. Gene 0317 is increased 5.4-fold on barley $\beta$-glucan ( $p$-value $<0.0002$ ) and gene 1149 is increased 9.4-fold on starch ( $p$-value $<0.037$ ) relative to growth on glucose

Table 2 Orthologs of translated sequences encoded by candidate genes from Pjdr2

\begin{tabular}{|c|c|c|c|}
\hline $\mathrm{LT}^{\mathrm{a}}$ & Protein & Orthologue (UniProt accession) & Identity $(\%)^{k}$ \\
\hline 0951 & multimodular $\mathrm{Bg} \mid 16 \mathrm{~A}_{1}$ & laminarinase from Thermotoga maritima (Q9WXN1) & 34 \\
\hline 0952 & $\mathrm{Bgl} 16 \mathrm{~A}_{2}$ & probable lichinase from Bacillus subtilis (P04957) & 71 \\
\hline 0774 & Amy $13 \mathrm{~A}_{1}$ & extracellular amylase from Bacillus megaterium (P20845) & 46 \\
\hline 5200 & multimodular Amy13 $\mathrm{A}_{2}$ & amylopullanase from Thermoanaerobacter pseudethanolicus (P38939) & 33 \\
\hline 0783 & Amy $13 \mathrm{~A}_{3}$ & intracellular maltogenic amylase from B. subtilis (O06988) & 47 \\
\hline 0771 & $\mathrm{SBP}$ & maltodextrin binding protein from Bacillus subtilis 168 (O06989) & 33 \\
\hline 1340 & SYM $^{d}$ & AraE xylose and arabinose symporter in B. subtilis (X98354) & 49 \\
\hline
\end{tabular}

a LT, locus tag annotated as Pjdr2_\#\#\#\# abbreviated to only consist of the numeric portion, \#\#\#\#

bamino acid sequence identity

'SBP, solute binding protein

${ }^{\mathrm{d}}$ SYM, symporter 
Table 3 Expression analysis of genes encoding ABC transporters during growth on barley $\beta$-glucan, starch, cellobiose and maltose

\begin{tabular}{|c|c|c|c|c|c|c|c|c|c|c|c|c|}
\hline \multirow[t]{2}{*}{$\mathrm{LT}^{\mathrm{a}}$} & \multirow[t]{2}{*}{ Protein product ${ }^{b}$} & \multicolumn{5}{|c|}{ Fold change ${ }^{c}$} & \multicolumn{6}{|c|}{ Linear RPKM Values $^{d}$} \\
\hline & & $\mathrm{B} / \mathrm{YE}$ & C/YE & $\mathrm{G} / \mathrm{YE}$ & $S / Y E$ & $M / Y E$ & $\mathrm{~B}$ & C & G & $S$ & M & YE \\
\hline 0472 & BPD transport system IMP & NS & $\underline{23.1}$ & $\underline{18.3}$ & NS & $\underline{2.7^{*}}$ & 1.7 & 27.7 & 22 & 1.1 & 3.3 & 1.2 \\
\hline 0473 & BPD transport system IMP & NS & $\underline{20.8}$ & $\underline{18.9}$ & NS & $\underline{3}$ & 1.8 & 31 & 28.2 & 1.9 & 4.4 & 1.5 \\
\hline 0474 & extracellular SBP & 0.7 & $\underline{7.4}$ & $\underline{9.6}$ & NS & NS & 6.5 & 71.7 & 92.8 & 8.6 & 13.4 & 9.7 \\
\hline 0728 & extracellular SBP & 0.3 & $\underline{7.2}$ & 0.2 & NS & 0.2 & 7.1 & 173.8 & 4 & 19 & 6 & 24 \\
\hline 0729 & BPD transport system IMP & 0.4 & $\underline{8.6}$ & 0.5 & NS & 0.4 & 6.2 & 119.1 & 6.5 & 12.8 & 5.1 & 13.9 \\
\hline 0730 & BPD transport system IMP & 0.5 & $\underline{8.8}$ & 0.5 & NS & 0.5 & 7.3 & 134.5 & 8.2 & 16.4 & 7.7 & 15.2 \\
\hline 0771 & extracellular SBP & 0.1 & 0 & 0 & 86.4 & $\underline{24.1}$ & 3.9 & 2.4 & 2.6 & 5524 & 1543.1 & 64 \\
\hline 0772 & BPD transport system IMP & 0.1 & 0.1 & 0.1 & $\underline{94.3}$ & 34.7 & 1.1 & 0.8 & 0.9 & 1174 & 432.6 & 12.5 \\
\hline 0773 & BPD transport system IMP & 0.1 & 0.1 & 0.1 & 134.8 & $\underline{58.3}$ & 1.3 & 0.8 & 0.8 & 1390 & 600.5 & 10.3 \\
\hline 0949 & BPD transport system IMP & 1451.2 & NS & NS & NS & NS & 3602 & 2.1 & 2 & 2.2 & 3.1 & 2.5 \\
\hline 0950 & BPD transport system IMP & 1498.5 & 1.7 & NS & NS & NS & 5522 & 6.4 & 3.5 & 4.8 & 4.9 & 3.7 \\
\hline 0953 & extracellular SBP & 1220.8 & $\underline{2}$ & NS & NS & 0.6 & 5381 & 8.8 & 3.5 & 5 & 2.6 & 4.4 \\
\hline 1320 & extracellular SBP & $0.2^{*}$ & 0.6 & 0.1 & $0.4^{*}$ & 0.1 & 4.2 & 11.5 & 1.4 & 6.9 & 1.8 & 18.6 \\
\hline 1321 & BPD transport system IMP & 0.1 & NS & 0.1 & $0.6^{*}$ & 0.1 & 0.7 & 4.8 & 0.5 & 3 & 0.5 & 5 \\
\hline 1322 & BPD transport system IMP & 15.1 & NS & 0.3 & 0.6 & 0.4 & 111.3 & 8.1 & 2.6 & 4.6 & 3.2 & 7.4 \\
\hline 3245 & periplasmic binding protein & $\underline{3.5}$ & $\underline{2.6}$ & $\underline{2.3}$ & $\underline{2.3}$ & $\underline{10.9}$ & 158.4 & 119.1 & 103.9 & 107 & 500.4 & 45.7 \\
\hline 3597 & extracellular SBP & $\underline{15}$ & 0.5 & $0.6^{*}$ & $\underline{2.4^{*}}$ & NS & 19.9 & 0.7 & 0.8 & 3.2 & 1.1 & 1.3 \\
\hline 5314 & BPD transport system IMP & 39.9 & 0.4 & 0.2 & 0.5 & 0.3 & 426.6 & 4.4 & 2.1 & 5.8 & 2.7 & 10.7 \\
\hline 5315 & BPD transport system IMP & 42.1 & 0.4 & 0.2 & $0.4^{*}$ & 0.2 & 379.7 & 3.2 & 1.4 & 3.7 & 1.4 & 9 \\
\hline 5316 & extracellular SBP & $\underline{31.1}$ & 0.2 & 0.1 & 0.3 & 0.1 & 797.5 & 5.4 & 1.9 & 7.5 & 1.6 & 25.7 \\
\hline 5589 & extracellular SBP & 0.5 & 0.5 & $0.5^{*}$ & NS & $\underline{9.8}$ & 2.2 & 2.1 & 2.3 & 3.3 & 42.9 & 4.4 \\
\hline 5590 & BPD transport system IMP & NS & NS & NS & NS & $\underline{10.9}$ & 1.1 & 1.2 & 1.8 & 1.4 & 15.3 & 1.4 \\
\hline 5591 & BPD transport system IMP & 0.5 & NS & NS & NS & 14.9 & 0.4 & 0.6 & 0.9 & 1.1 & 13.7 & 0.9 \\
\hline 5596 & BPD transport system IMP & 3.1 & $\underline{44.8}$ & 0.6 & 0.6 & 0.4 & 19.7 & 281.7 & 3.5 & 3.5 & 2.4 & 6.3 \\
\hline 5597 & BPD transport system IMP & 3.3 & $\underline{52.2}$ & $0.5^{*}$ & NS & 0.2 & 14.1 & 219.5 & 2.1 & 2.2 & 1 & 4.2 \\
\hline 5598 & extracellular SBP & $\underline{4.4}$ & $\underline{54.5}$ & 0.1 & $0.2^{*}$ & 0.1 & 40.3 & 494.9 & 1.1 & 2.3 & 0.9 & 9.1 \\
\hline 5960 & BPD transport system IMP & $\underline{15.3}$ & $\underline{154.6}$ & $\underline{15.2}$ & NS & 0.5 & 269.7 & 2721 & 267.6 & 22.6 & 8.8 & 17.6 \\
\hline 5961 & BPD transport system IMP & $\underline{16}$ & 145.5 & 12.7 & NS & 0.5 & 227.1 & 2062 & 179.8 & 22.6 & 7.8 & 14.2 \\
\hline 5962 & extracellular SBP & 18.1 & 118.5 & 12.2 & NS & 0.3 & 620.6 & 4070 & 419.6 & 33.1 & 9.4 & 34.3 \\
\hline
\end{tabular}

aT, locus tag annotated as Pjdr2_\#\#\#\# abbreviated to consist only of the numeric portion, \#\#\#

${ }^{\mathrm{b}} \mathrm{SBP}$, solute binding protein; IMP, inner membrane protein; BPD, binding protein dependent

'Transcript levels of candidate genes that were expressed 2-fold greater (underlined) and those that were expressed 4-fold greater (bold) than the yeast extract without carbohydrate are indicated. The growth substrates are shown as follows: B, barley $\beta$-glucan; $C$, cellobiose; $G$, glucose; $S$, starch; $M$, maltose; $Y E$, yeast extract. Significance of fold change data is judged by having a $p$-value no more than 0.01 . Data with $p$-values between 0.01 and 0.05 are denoted with an asterisk, and those with $p$-values greater than 0.05 are designated as not significant (NS)

${ }^{d}$ RPKM values are defined as Reads Per Kilobase per Million reads sequenced

Cellobiose as a growth substrate results in increased expression of several genes encoding putative ABC transporters in Pjdr2. The genes 5960, 5961 and 5962 show greater than 118-fold increase in expression on cellobiose relative to YE. Both glucose and barley $\beta$-glucan also induce the genes encoding this transporter although to a lower extent (Table 3). From the transcriptome data it is not known precisely how cellobiose is converted to glucose for entry into glycolysis. As detailed above for the intracellular processing of glucooligosaccharides resulting from barley $\beta$-glucan utilization the putative intracellular
GH3 $\beta$-glucosidase (gene 0317) may serve this role. This gene is expressed on cellobiose at nearly the same increased level as found with growth on barley $\beta$-glucan relative to other sugars, but not YE (Table 1). In addition, gene 0750 encoding a putative intracellular $\beta$-xylosidase $\left(\mathrm{Xyn}_{\left.43 \mathrm{~B}_{2}\right)}\right.$ that was earlier predicted to be involved in $\mathrm{xy}$ lan utilization due to its 100 -fold increase on xylan relative to YE (Additional file 1) is found in this work to be increased 177-fold during growth on cellobiose [1] (Table 1). This gene may encode the enzyme primarily responsible for hydrolysis of cellobiose. If so, this putative xylosidase 
either has dual substrate specificity or it actually encodes a GH43 $\beta$-glucosidase the expression of which is induced by cellobiose and to a lesser extent xylobiose. The GH43 family does not as yet contain an enzyme with a reported $\beta$-glucosidase activity. The expression of $x y n 43 B_{2}$ is also increased on barley $\beta$-glucan by 3 -fold (Table 1 ) relative to YE and may contribute as well to the hydrolysis of the glucooligosaccharides derived from this polymer.

\section{Genes involved in starch utilization}

Pjdr2 grows very efficiently on starch [17]. Utilization of this $\alpha$-1,4-linked glucose storage polysaccharide appears similar to barley $\beta$-glucan as this polysaccharide is also chemically simple relative to xylans with fewer enzymes required for degradation to glucose. The genome of Pjdr2 encodes four GH13 amylases. Three of these, Amy $13 A_{1}, A m y 13 A_{2}$, and Amy $13 A_{3}$ have significantly increased transcript levels ranging from 55-fold to over 100-fold increased expression during growth on starch (Table 1). Both Amy13A $\mathrm{A}_{1}$ (gene 0774) and Amy $13 \mathrm{~A}_{2}$ (gene 5200) are predicted to be secreted and primarily responsible for endo-hydrolysis of native starch. The amy $13 A_{2}$ gene encodes a large multimodular enzyme including SLH domains and CBM's for cell surface proximal substrate localization (Fig. 4) while amy13A encodes only a catalytic domain. The starch utilization system in Pjdr2 also has a predicted intracellular amylase, $\mathrm{Amy}_{13 \mathrm{~A}_{3}}$ (gene 0783) presumably to complete the degradation of the transported, intracellular maltodextrins.

Amy13A 1 shares $46 \%$ amino acid sequence identity with the extracellular amylase from Bacillus megaterium (UniProt accession: P20845) (Table 2) [27, 28] and Amy $13 \mathrm{~A}_{2}$ shares $33 \%$ identity over a large portion of its modular sequence with an amylopullanase from Thermoanaerobacter pseudethanolicus (UniProt accession: P38939) [29]. Amy13A 3 shares $47 \%$ amino acid identity with an intracellular maltogenic amylase from $B$. subtilis (UniProt accession: O06988) [30] where it is thought to function in the conversion of maltotriose and larger maltodextrins to maltose and glucose (Table 2).

A single $A B C$ transporter gene cassette showed increased transcript levels during growth on starch relative to YE (Table 3). The genes for this transporter (genes 0771, 0772 and 0773) are just upstream of amy13 $A_{1}$ (0774) and form a predicted operon (Fig. 1c). The solute binding protein (gene 0771) of this transporter shares $33 \%$ amino acid identity with a maltodextrin binding protein from Bacillus subtilis 168 (Table 2) [31]. This putative maltose/maltodextrin $\mathrm{ABC}$ transporter gene cassette was shown to be markedly up-regulated during growth on xylans (Fig. 3b, Additional file 2) [1]. However, the genomic localization of this $\mathrm{ABC}$ gene cluster within a predicted operon containing the gene encoding extracellular amylase suggests its primary function is that of a maltodextrin transporter (Fig. 1c). This overlap in regulation will be further discussed below.

The high amino acid sequence identity between Amy $13 \mathrm{~A}_{3}$ and the maltogenic amylase from $B$. subtilis suggest that this enzyme might process transported maltodextrins to glucose and maltose [30]. As a component of a complete starch utilization system, gene 1149 encodes a putative $\alpha$-glucan phosphorylase (MalP) allowing for phosphorolytic cleavage of intracellular maltose $[32,33]$. Expression levels of this gene on starch compared to YE yielded insignificant results ( $p$-value, 0.403 ), but a 9.1-fold transcript increase is observed relative to growth on glucose ( $p$-value, 0.004) (Table 1). Transcript data for the fourth predicted amylase encoding gene, 1045, was not considered statistically significant ( $p$-value, 0.635$)$ and did not appear to exhibit dynamic regulation on starch, barley $\beta$-glucan or xylan and linear RPKM values were comparatively low (1.2-1.6).

\section{Genes with increased expression during growth on maltose}

As the primary hydrolysis limit product of starch, maltose was included in this study to distinguish physiological features for efficient starch utilization. The transporter genes described above as part of the putative starch utilization operon are also upregulated. In addition, genes 5589, 5590 and 5591 encoding a second $\mathrm{ABC}$ transporter are up-regulated approximately 10-fold on maltose over YE (Table 3). Once internalized, maltose would be expected to follow the pathway similar to that predicted in starch utilization; however, for this growth condition expression of the gene encoding the MalP protein is not increased relative to any other growth condition.

This finding reveals a difference between the intracellular processing of maltodextrins derived from starch hydrolysis by the surface localized multimodular Amy $13 \mathrm{~A}_{2}$ and maltose directly assimilated. A focused search failed to identify homologs of genes known for the conversion of maltose or maltose-6-phosphase (e. g. glucose phosphorylase or 6-phospho-alpha-glucosidase). Two other genes upstream of those encoding the maltose specific transporter identified above code for proteins annotated as an oxidoreductase (gene 5587) and a hypothetical protein (gene 5588) and the predicted operon appears related to the $t h u A B$ encoding operon involved in trehalose utilization in Agrobacterium tumefaciens [34] (Table 1). This suggests that Pjdr2 converts maltose to 3-keto-maltose.

\section{Monosaccharide assimilation and metabolism}

From genome analysis [5], intracellular metabolism of the hexose, glucose, and the pentoses, xylose and arabinose, are expected to follow through the Embden-Meyerhof-Parnas (EMP) pathway and pentose phosphate pathway (PPP), respectively, for entrance into the tricarboxylic acid (TCA) 
cycle. Following transport of arabinose through the previously identified arabinose responsive $\mathrm{ABC}$ transporter [1], this sugar may be converted to ribulose- 5 -phosphate by the arabinose isomerase and ribulose kinase enzymes. In Pjdr2, the gene 2502 (Table 4) attains a 24-fold increase in transcript level with growth on arabinose and 4.9-fold on sorghum MeGAX $_{n}$ (Additional file 2). Based on transcript levels the candidate ribulose kinase enzyme is encoded by gene 4209 . This enzyme is a distant homolog ( 21 \% ID) to the AraB protein which is a component of the L-arabinan utilization system of Geobacillus stearothermophilus [35] (Table 2). Transcript levels of gene 4209 are increased 17-fold on arabinose (Table 4) and 3-fold on sorghum MeGAX $_{\mathrm{n}}$ relative to YE (Additional file 2). This gene does not show an increased transcript level on other carbohydrate growth conditions used in this study. The genes 0977, 0978 and 0979 encoding an $\mathrm{ABC}$ transporter are primarily responsive to xylose resulting in an average of 135-fold increase in transcript level relative to YE (Table 4). These genes also showed significant but much lower fold increases on glucose and arabinose. Additionally, a predicted symporter encoded by gene 1340 shares $49 \%$ identity with the AraE xylose and arabinose symporter in B. subtilis (Table 2) [36]. Expression of this gene is responsive to xylose resulting in a 162-fold increase. This gene is also expressed on cellobiose (Additional file 2), glucose and arabinose although to a much lower extent than observed on xylose (Table 4). Conversion of xylose to xylulose-5-phosphate follows a similar path as arabinose since genes encoding xylose isomerase (gene 5159) and xylulose kinase (gene 5158) result in nearly a 100 -fold and 63-fold increase in expression, respectively, on xylose compared to YE controls (Table 4). Growth on xylans also resulted in transcript increases of 35 -fold and greater for these two genes (Additional file 2).

While the genes that encode the transporters that import xylose and arabinose can be identified based on homology and increased transcript levels, a system for efficient glucose assimilation is less apparent. Genes encoding three putative $\mathrm{ABC}$ transporters showed

Table 4 Regulation of genes involved in monosaccharide transport and introduction into metabolic pathways

\begin{tabular}{|c|c|c|c|c|c|c|c|c|}
\hline \multirow[t]{2}{*}{$\mathrm{LT}^{\mathrm{a}}$} & \multirow[t]{2}{*}{ Protein product ${ }^{b}$} & \multicolumn{3}{|c|}{ Fold change ${ }^{c}$} & \multicolumn{4}{|c|}{ Linear RPKM Values $^{d}$} \\
\hline & & G/YE & $X / Y E$ & AYYE & $\bar{G}$ & $x$ & A & YE \\
\hline \multicolumn{9}{|c|}{ Monosaccharide metabolism } \\
\hline 0170 & glucokinase & NS & NS & NS & 119.2 & 140.7 & 131.2 & 128.5 \\
\hline 2502 & arabinose isomerase & 0.5 & 0.6 & $\underline{23.8}$ & 81.6 & 96.3 & 4117.9 & 173.0 \\
\hline 4209 & ribulose kinase & 0.6 & NS & $\underline{17.1}$ & 13.0 & 20.9 & 369.6 & 21.7 \\
\hline 5159 & xylose isomerase & 0.4 & $\underline{99.9}$ & 0.5 & 21.3 & 6046.1 & 30.9 & 60.5 \\
\hline 5158 & xylulose kinase & 0.4 & $\underline{62.9}$ & $\underline{19.0}$ & 16.9 & 2951.2 & 19.0 & 46.9 \\
\hline \multicolumn{9}{|c|}{ Monosaccharide transporters } \\
\hline 0472 & BPD transport system IMP & $\underline{18.3}$ & $\underline{6.4}$ & $\underline{16.8}$ & 22 & 7.7 & 20.2 & 1.2 \\
\hline 0473 & BPD transport system IMP & $\underline{18.9}$ & $\underline{6.0}$ & 13.9 & 28.2 & 9.0 & 20.8 & 1.5 \\
\hline 0474 & extracellular SBP & $\underline{9.6}$ & $\underline{3.1}$ & $\underline{5.6}$ & 92.8 & 30.4 & 54.2 & 9.7 \\
\hline 0661 & extracellular SBP & $0.4^{*}$ & NS & $\underline{1917.4}$ & 1.4 & 4.8 & 7460.5 & 3.9 \\
\hline 0662 & NBD & NS & NS & $\underline{1267.6}$ & 1.0 & 2.1 & 3374.4 & 2.7 \\
\hline 0663 & BPD transport system IMP & 0.2 & 0.6 & $\underline{1301.2}$ & 0.8 & 2.0 & 4343.5 & 3.3 \\
\hline 0977 & extracellular SBP & $\underline{16.8}$ & 190.6 & $\underline{8.3}$ & 154.2 & 1747.9 & 76.2 & 9.2 \\
\hline 0978 & NBD & 15.2 & 102.1 & $\underline{5.9}$ & 80.2 & 538.0 & 30.9 & 5.3 \\
\hline 0979 & BPD transport system IMP & $\underline{17.6}$ & 111.9 & $\underline{6.4}$ & 105.9 & 671.5 & 38.7 & 6.0 \\
\hline 1340 & symporter & $\underline{39.1}$ & $\underline{162.0}$ & $\underline{14.0}$ & 120.5 & 499.9 & 43.4 & 3.1 \\
\hline 2400 & extracellular SBP & $\underline{4.8}$ & 3.7 & 3.8 & 244.9 & 188.0 & 193.4 & 51.1 \\
\hline 2401 & NBD & $\underline{4.3}$ & 1.9 & $\underline{2.3}$ & 93.8 & 41.5 & 49.4 & 21.9 \\
\hline 2402 & BPD transport system IMP & $\underline{4.8}$ & 3.3 & 3.5 & 116.1 & 79.2 & 84.0 & 24.0 \\
\hline
\end{tabular}

a $\mathrm{LT}$, locus tag annotated as Pjdr2 \#\#\#\# abbreviated to consist only of the numeric portion, \#\#\#

${ }^{b} S B P$, solute binding protein; IMP, inner membrane protein; BPD, binding protein dependent; NBD, nucleotide binding domain

'Transcript levels of candidate genes that were expressed 2-fold greater (underlined) and those that were expressed 4-fold greater (bold) than the yeast extract without carbohydrate are indicated. The growth substrates are shown as follows: G, glucose; $X$, xylose; $A$, arabinose; $Y E$, yeast extract. Significance of fold change data is judged by having a $p$-value no more than 0.01 . Data with $p$-values between 0.01 and 0.05 are denoted with an asterisk, and those with $p$-values greater than 0.05 are designated as not significant (NS)

${ }^{\mathrm{d}} \mathrm{RPKM}$ values are defined as Reads Per Kilobase per Million reads sequenced 
increased transcript levels with growth on glucose, but for only two of these ABC transporters (genes 0472, 0473 and 0474 and genes 2400, 2401 and 2402) is it possible that glucose may be the target sugar for transport. For both of these transporter gene sets transcript is increased not only on glucose, but also similarly increased on arabinose, xylose and cellobiose (Tables 3 and 4). The 0472-0474 gene set is increased more significantly at approximately 20 -fold relative to the YE control, while the 2400-2402 gene set is just greater than the significance cutoff of 4-fold (Table 4 and Additional file 2). The third $\mathrm{ABC}$ transporter gene set whose transcript is significantly increased with growth on glucose (genes 0977, 0978 and 0979) is assigned as a xylose transporter. While its expression is significant with growth on glucose, it is very low relative to growth on xylose.

Analysis for phosphotransferase systems (PTS) reveals two operons (gene sets 2007-2010 and 62216226) encoding all protein components of a complete PTS system. Based on homology, the 6221-6226 gene set appears very likely to be a mannitol transport system, while the 2007-2010 set encodes a EIIA component (gene 2010) which is annotated as a glucose superfamily transporter, and a separate protein product (gene 2009) encoding the EIIBC components annotated as an $\mathrm{N}$-acetylglucosamine specific transporter (Additional file 2). None of the genes encoding the complete PTS system components have increased transcript responsive to growth on glucose relative to YE. Interestingly, two unlinked PTS system components (gene 3804 annotated as an Enzyme I complex and gene 0174 annotated as HPr phosphocarrier protein) have relatively high constitutive expression levels, but their roles are unclear (Additional file 2). The analysis for potential glucose specific transporters is not conclusive from this data. Once transported into the cell, conversion of glucose to glucose-6-phosphate for entry into glycolysis appears to be mediated by only a single enzyme: a glucokinase (gene 0170) which yields an average RPKM value of $128 \pm 15$ over all the tested growth conditions (Table 4). This physiological data underscores original research which showed that Pjdr2 does not efficiently utilize simple sugars in minimal salt media [2].

Overlapping regulation: starch $>$ xylan $>$ soluble $\beta$-glucan Unexpectedly, the combined data for barley $\beta$-glucan, starch and xylan reveals a regulatory connection for utilization of these polymers. This can be seen in quantitative comparisons of the expression of genes encoding the secreted multimodular GH16, GH13, and GH10 endolytic enzymes and those encoding their associated substrate binding proteins that serve as a representative of the specific $A B C$ transporter for the saccharides generated by these enzymes on the cell surface (Fig. 2). Growth on xylans, both $\mathrm{GX}_{\mathrm{n}}$ and $\mathrm{GAX}_{\mathrm{n}}$, supports the enhanced expression of genes associated with utilization of xylans and starch but not those associated with the utilization of soluble $\beta$-glucan. While barley $\beta$-glucan induces genes related to its extracellular degradation and assimilation, these results show that it also induces 8 of the 13 glycoside hydrolase genes involved in xylan utilization (Table 1) [1]. Furthermore, while growth on xylan does not induce any soluble $\beta$-glucan utilization genes it does induce genes encoding all of the GH13

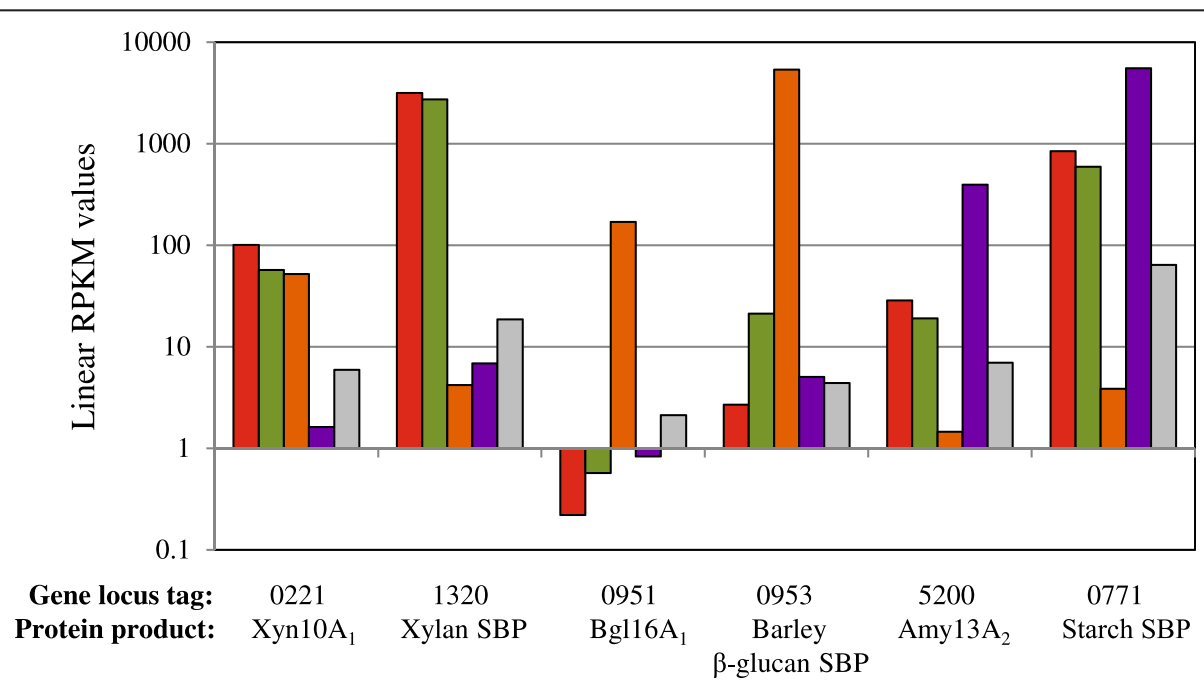

Fig. 2 Comparison of gene expression of surface localized substrate specific glycoside hydrolases and their regulon associated solute binding protein. RPKM (Reads Per Kilobase per Million reads sequenced) values from transcriptomic studies following Pjdr2 growth on sweetgum GXn (SG), sorghum GAXn (SO), barley $\beta$-glucan (B) or starch (S) for the genes encoding the large multimodular surface anchored glycoside hydrolase and ABC transporter solute binding protein (SBP) which represents regulation for each of the three polysaccharides. A culture containing only $0.5 \%$ yeast extract without carbohydrate (YE) served as control for comparison. Locus tag annotated as Pjdr2_\#\#\#\# abbreviated to only consist of the numeric portion, \#\#\#\# 
$\alpha$-amylases and the $\mathrm{ABC}$ transporter considered to be involved in starch depolymerization and transport for utilization (Fig. 3b and Additional file 2) [1] with the exception of the putative $\alpha$-glucan phosphorylase gene, malP. Following growth on starch, Pjdr2 does not induce genes for either xylan or barley $\beta$-glucan utilization. These relationships are represented in the heat map shown in Fig. $3 b$ in which expression of genes encoding ABC transporter proteins as well as accessory enzymes for intracellular metabolism of assimilated oligosaccharides are shown. These findings may be due to a metabolic substrate preference in a manner similar to glucose mediated catabolite repression, or result from evolved enzyme systems for utilization of polysaccharides that are typically associated. In cereal grains, these three carbohydrates can be found together, with xylan and $\beta$-glucan localized more to the cell wall and outer layers, and the starch consolidated in the endoplasm [37]. The model that currently describes this relationship (Fig. 3a) can be described as starch first, xylan second and barley $\beta$-glucan third. From the observed coordinate gene expression, Pjdr2 appears prepared to utilize multiple polysaccharides (Fig. 3b).
In consideration of the expression of the genes encoding intracellular xylanases, e.g. Xyn43B ${ }_{1}$ and Xyn8 [1] on barley $\beta$-glucan, it is possible that these enzymes may have a bifunctional role in the intracellular hydrolysis of $\beta$-glucooligosaccharides, thereby, providing an additional route for the intracellular processing of the barley $\beta$-glucan derived glucooligosaccharides and cellobiose.

Other genes involved in transport also show overlapping regulation. The predicted $\mathrm{ABC}$ transporter previously annotated as a "multiple sugar transport system" consisting of the genes 5314, 5315 and 5316 is shown to have increased expression on barley $\beta$-glucan (Table 3 ) similar to that observed for xylan [1]. This is the only $A B C$ transporter gene set that follows the pattern of expression during growth on barley $\beta$-glucan as that observed for the xylan specific glycoside hydrolase genes (Fig. 3b), supporting the possibility that it might be bifunctional in substrate recognition. One other gene encoding an $\mathrm{ABC}$ transporter component also follows this pattern. Gene 1322 (Table 3) encoding an inner membrane component, UgpE (BPD transport system IMP, Fig. 1a), of the aldouronate utilization gene cluster $[1,4]$ has a markedly increased transcript level (15-fold) on barley

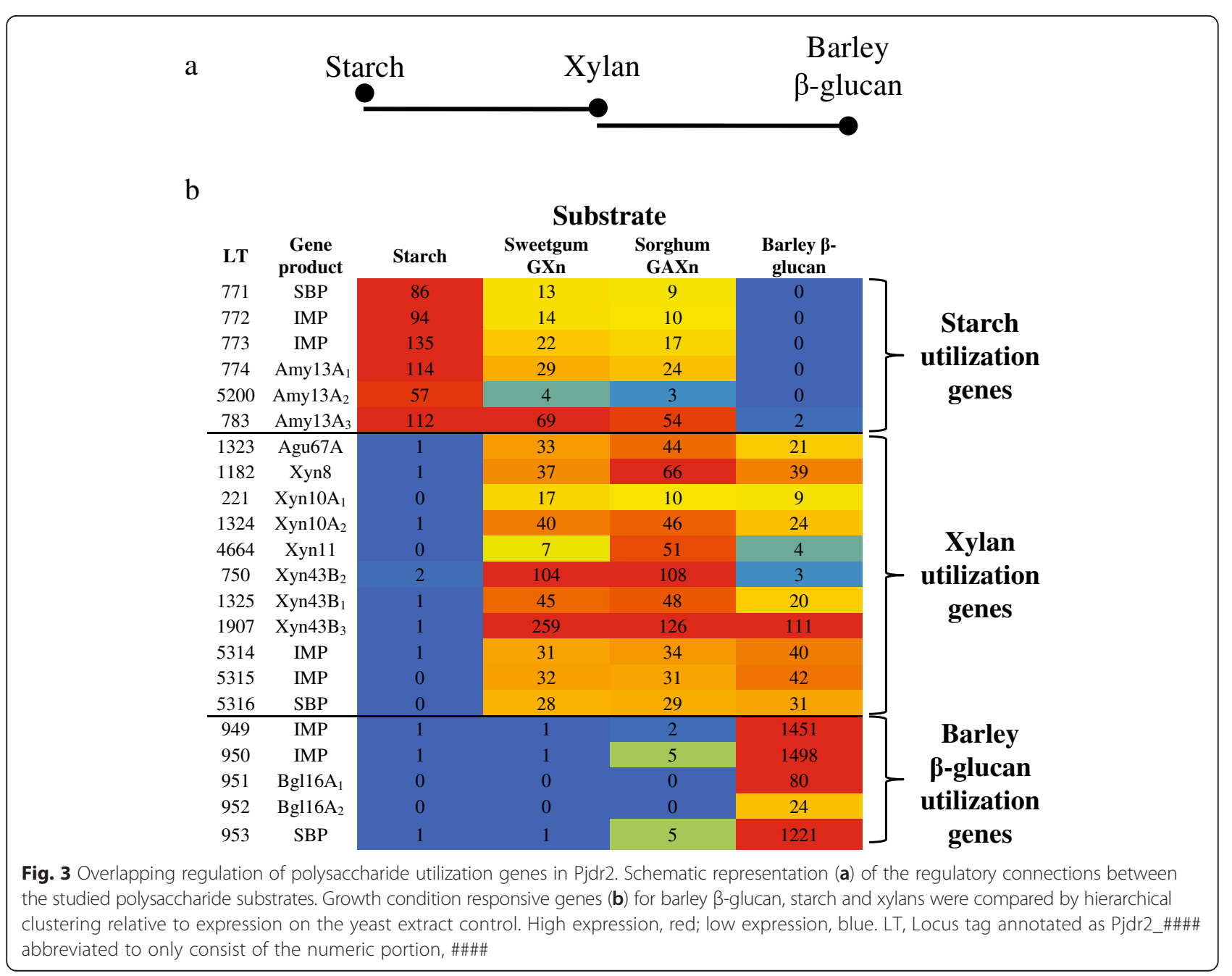


$\beta$-glucan. Studies are underway to elucidate these overlapping regulatory connections.

\section{Overlapping regulation: cellobiose and xylobiose}

Some genes with increased transcript levels during growth on cellobiose were found to also have increased expression levels with growth on xylans. The gene cluster 5596, 5597 and 5598 (Table 3) encodes an ABC transporter annotated as an "unknown carbohydrate transporter" and has been assigned a potential role in xylan utilization based on increased transcript levels (Additional file 1) [1]. Analysis of growth on cellobiose indicates these genes are expressed at a level comparable to that on xylan. A corollary to this finding is the observation that growth on cellobiose also resulted in increased transcript levels for genes 0728, 0729 and 0730 encoding an $\mathrm{ABC}$ transporter previously assigned a putative function in xylooligosaccharide $\left(X_{2}\right.$ and $\left.X_{3}\right)$ transport [1]. From the similar level of expression on both xylan and cellobiose, it is proposed that these transporters may be specific for disaccharides such as cellobiose and the primary neutral product of enzymatic xylan hydrolysis, xylobiose. These findings indicate that the transporters of $\beta$-configured oligosaccharides may be promiscuous in their substrate recognition.

\section{Proteins with SLH domains}

Enzyme systems utilized by Pjdr2 for the extracellular processing of xylan, barley $\beta$-glucan and starch share a common theme. These systems include extracellular cellassociated multimodular glycoside hydrolases to generate oligosaccharides that are released in close proximity to the bacterial cell wall. This functionality is mediated by surface layer homology (SLH) domains that anchor the enzymes to the cell surface $[2,38,39]$ and carbohydrate binding modules $(\mathrm{CBM})$ that presumably associate the enzyme with the target polysaccharide [40]. These cellsurface proximal oligosaccharides are then efficiently transported with substrate specific $A B C$ transporters and further hydrolyzed within the cell to monosaccharides for introduction into catabolism. This surface localization represents a strategy for competitive utilization of these polysaccharides. As part of this work we sought to define the roles of SLH domains in Pjdr2 in the processing of plant polysaccharides.

In total, there are 77 genes encoding proteins with regions homologous with SLH domains. Of these, 73 have two or more consecutive SLH domains which is the minimum set thought to be required for tight binding to the cell wall [41]. Of the 77 SLH domain containing proteins, 29 are predicted to be involved in carbohydrate processing (Table 5) as indicated through domain analysis. In this smaller set, the average calculated protein size is nearly $193 \mathrm{kDa}$ and the average predicted $\mathrm{pI}$ is 4.74 with a standard deviation of just 0.10 . Domain and BLASTp analysis (Table 5) shows the diversity of functions of associated carbohydrate active enzymes among these SLH proteins (Table 5). In the current transcriptomic data set, only a single SLH-bearing gene has been identified being involved in the catalysis of either xylan (gene 0221), barley $\beta$-glucan (gene 0951) or starch (gene 5200) utilization from the 29 identified SLH-encoding carbohydrate processing genes (Table 5). Of the other SLH domain containing proteins in this list it can be seen that Pjdr2 may utilize numerous other polysaccharides with the same strategy. Some of these include arabinan, galactomannan, chitin, pectin and hyaluronan (Fig. 4).

\section{Polysaccharide utilization in Pjdr2}

From the studies presented here for the utilization of barley-derived $\beta$-glucan and starch, we observe a similar strategy evolved by Pjdr2 as illustrated in the earlier xylan transcriptome report [1]. In each case coordinately expressed gene sets have been identified (Fig. 1) and central to each encoded enzyme system is a multimodular glycoside hydrolase containing carbohydrate binding modules which afford interaction with polysaccharide substrates and a triplicate set of SLH domains for cell surface localized formation of oligosaccharides (Fig. 4).

For processing of $\beta-1,3(4)$-glucans, contiguous genes encoding transcriptional regulators, $\mathrm{ABC}$ transporters, the multimodular cell-associated Bgl16A 1 and the secreted non-modular $\mathrm{Bgl}_{16 \mathrm{~A}_{2}}$ catalytic domain along with an associated $\mathrm{ABC}$ transporter comprise a $\beta$-glucan utilization regulon. In this case both secreted enzymes digest barley $\beta$-glucan to tri-, tetra-, penta- and hexasaccharides, and laminarin to mono-, di-, tri- and tetrasaccharides indicating similar functions for both enzymes [18]. These oligosaccharides resulting from extracellular barley $\beta$-glucan hydrolysis and cellobiose (from either barley $\beta$-glucan or growth on cellobiose) are presumably transported into the cell where they are subsequently degraded to monosaccharides by the action of a $\mathrm{GH} 3$ endoglucanase and/or a novel GH43 enzyme $\left(\mathrm{Xyn}_{43 \mathrm{~B}_{2}}\right)$ with $\beta$-glucosidase functionality.

For starch processing, a regulon encoding a putative maltodextrin $\mathrm{ABC}$ transporter together with the nonmodular Amy $13 \mathrm{~A}_{1}$ managed starch utilization. Encoded distally, the multimodular cell-associated amylase Amy $13 \mathrm{~A}_{2}$ likely produces small maltodextrins proximal to the cell surface. These may then be taken up and processed by intracellular maltogenic Amy $13 \mathrm{~A}_{3}$ to yield maltose and glucose. Final conversion of maltose is thought to occur through the action of an $\alpha$-glucan phosphorylase yielding glucose and glucose-1 phosphate.

For the soluble $\beta$-glucan, starch and xylan utilization systems, two endo-acting hydrolases may work synergistically with each other for efficient depolymerization of the specific polymeric substrate to oligosaccharides. The modular property of the larger enzyme allows 
Table 5 List of surface layer homology domain containing proteins of Pjdr2 proposed to be involved in extracellular polysaccharide processing

\begin{tabular}{|c|c|c|c|c|c|}
\hline $\mathrm{LT}^{\mathrm{a}}$ & Domain architecture $e^{b, c, d}$ & Secretion $^{f}$ & $\mathrm{MW}(\mathrm{kDa})^{\mathrm{g}}$ & $\mathrm{pl}^{\mathrm{g}}$ & Putative function ${ }^{\mathrm{h}}$ \\
\hline $0221 / \mathrm{Xyn}_{10 A_{1}}$ & 3CBM4,9/GH10/CBM9/3SLH & Yes & 157 & 4.90 & $\beta$-xylanase \\
\hline 0680 & GH5/FN3/3CBM11/3SLH & Yes & 204 & 4.72 & $\beta$-mannanase \\
\hline 0824 & $\begin{array}{l}\text { 3SLH/GH16/3CBM4,9/DUF1533/ } \\
\text { GH16/3CBM4,9/DUF1533/CBM4,9/ } \\
\text { DUF1533/CF/DUF1533/CF }\end{array}$ & Yes & 313 & 4.65 & $\beta$-glucanase \\
\hline 0951/ Bgl16 $\mathrm{A}_{1}$ & 3SLH/GH16/2CBM4,9/CBM6/2CBM4,9 & Yes & 154 & 4.83 & $\beta$-glucanase \\
\hline 0964 & $\mathrm{RBT} / 2 \mathrm{CBM} 6 / \mathrm{RHB} / 3 \mathrm{SLH}$ & Yes & 228 & 4.81 & mycodextranase \\
\hline 1124 & $\mathrm{GH} 43 / \mathrm{CBM} 6 / 2 \mathrm{BIG} 2 / 2 \mathrm{SLH}$ & Yes & 159 & 4.71 & a-L-arabinofuranosidase \\
\hline 1125 & $\mathrm{GH} 20 / \mathrm{CBM}_{6} / \mathrm{BIG} 2 / 2 \mathrm{SLH}^{\mathrm{e}}$ & Yes & 213 & 4.75 & a-glucuronidase \\
\hline 1167 & DUF481/RHB/FN3/CBM9/FN3/3SLH & Yes & 185 & 4.79 & polysaccharide lyase \\
\hline 1173 & 2PL3/CBM9/3SLH & Yes & 210 & 4.81 & pectate lyase \\
\hline 1611 & $\mathrm{CCT} / \mathrm{PHP} / \mathrm{BIG} 3 / 3 \mathrm{SLH}$ & Yes & 230 & 4.90 & chitobiase (CCT/ESD) association \\
\hline 1796 & 2FN3/GH18/FN3/3SLH & Yes & 128 & 4.79 & chitinase \\
\hline 1997 & $\mathrm{CBM} 4,9 / \underline{\mathrm{AL} 3} / \mathrm{HP} / 3 \mathrm{SLH}$ & Yes & 229 & 4.90 & alginate lyase/heparinase \\
\hline 2544 & $2 \underline{N V S} / 2 F N 3 / 3 S L H$ & Yes & 170 & 4.99 & sialidase \\
\hline 3195 & $\underline{\mathrm{GAG}} / 3 \mathrm{SLH}$ & Yes & 153 & 4.85 & hyaluronate lyase \\
\hline 3554 & GH30/CBM6/CBM4,9/2FN3/BIG2/3SLH & Yes & 173 & 4.89 & endo-1,6-beta-glucosidase \\
\hline 3565 & 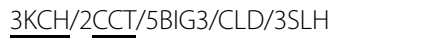 & Yes & 227 & 4.65 & CCT/ESD association \\
\hline 4054 & 2CBM6/PL3/RHB/3SLH & Yes & 237 & 4.75 & polysaccharide lyase \\
\hline 4093 & 3FN3/GH43/CBM6/3SLH & Yes & 200 & 4.70 & $\beta$-xylosidase \\
\hline 4104 & $\mathrm{GH} 27 / 3 \mathrm{CBM} \times 2 / 3 \mathrm{SLH}$ & Yes & 164 & 4.73 & a-galactosidase \\
\hline 4665 & GH59/CBM6/BIG4/BIG3/3SLH & Yes & 217 & 4.52 & $\beta$-galactosidase \\
\hline 4730 & GH53/BIG4/2CBM4,9/3SLH & Yes & 122 & 4.73 & $\begin{array}{l}\text { arabinogalactan } \\
\text { endo- } \beta-1,4 \text {-galactanase }\end{array}$ \\
\hline 5040 & GH66/5CBM6/AMY/3SLH & Yes & 182 & 4.83 & dextranase \\
\hline 5076 & $\underline{\mathrm{RHB}} / 2 \mathrm{CF} / \mathrm{FN} 3 / \mathrm{GH} 65 / 2 \mathrm{CF} / 3 \mathrm{SLH}$ & Yes & 273 & 4.77 & alpha-L-fucosidase \\
\hline 5200/ Amy $13 A_{1}$ & 4ESD / $/$ AMY/2FN3/3SLH & Yes & 235 & 4.75 & amylopullulanase \\
\hline 5272 & 2CBM11/BIG2/3SLH & Yes & 231 & 4.65 & carbohydrate binding \\
\hline 5379 & $3 \mathrm{SLH} / \underline{\mathrm{GH} 18}$ & Yes & 59 & 5.80 & peptidoglycan hydrolase \\
\hline 5534 & $\underline{\mathrm{RHB}} / 2 \mathrm{CRD} / 2 \mathrm{CLD} / \mathrm{BIG} 2 / 3 \mathrm{SLH}$ & Yes & 176 & 4.58 & polysaccharide lyase \\
\hline 5572 & $\mathrm{GH} 42 / 3 \mathrm{SLH}$ & Yes & 162 & 4.72 & $\beta$-galactosidase \\
\hline 6195 & 3PBX/3SLH & Yes & 77 & 4.53 & xylanase \\
\hline
\end{tabular}

aLT, locus tag annotated as Pjdr2_\#\#\#\# abbreviated to consist only of the numeric portion, \#\#\#\#. Surface anchored proteins directly involved in the utilization of xylan, barley $\beta$-glucan and starch are denoted in bold

${ }^{b}$ Domain predictions result from analysis of the proteins in the CCD (Conserved Domain Database) with an Expect Value threshold set to the default of 0.010 and increased to 0.10 to detect more divergent domains in unaccounted for regions or, as in some cases directly through the pfam database. Domain abbreviations are defined in order of appearance. CBM, Carbohydrate binding module; GH, Glycoside hydrolase; $S L H$, Surface layer homology domain; FN3, Fibronectin type 3 domain; DUF, Domain of unknown function; $C F$, Coagulation factor $5 / 8$ C-terminal domain; $R B T$, Ricin-type beta-trefoil; $R H B$, Right handed beta helix; $B / G$, Bacterial Ig-like domain; PL, Pectate lyase; CCT, Chitobiase/beta-hexosaminidase C-terminal domain in the early set domain superfamily; PHP, Polymerase and histidinol phosphatase domain; $A L$, Alginate lyase; $H P$, Heparinase; NVS, Non-viral sialidases; $G A G$, Glycosaminoglycan polysaccharide lyase family; $K C H$, Galactose oxidase central domain; CLD, Cadherin-like beta sandwich domain; AMY, Alpha amylase catalytic domain family; ESD, Early set domain; CPRD, Carboxypeptidase regulatory-like domain; PBX Putative bacterial xylanases

${ }^{C}$ For any given domain an abbreviation is provided as defined under superscript (b), with numbers preceding the abbreviation indicating the number of consecutive occurrences greater than one $(>1)$ of the domain and numbers following the abbreviation indicating the specific family of the detected domain, if any "In each modular protein the domain used to establish the "putative function" (sixth column) is underlined

${ }^{\mathrm{e} A n n o t a t e d}$ in the CAZy database as a GH115

fSecretion was deduced by detection of a signal peptide using the Signal-P server

${ }^{9}$ Molecular weight (MW) and isoelectric point (pl) predictions were obtained through the ProtParam tool available through the ExPASy web server

${ }^{h}$ Putative function is based either on the predicted function from domain assignment or a justification for assignment as a protein that is involved with sugar manipulations 


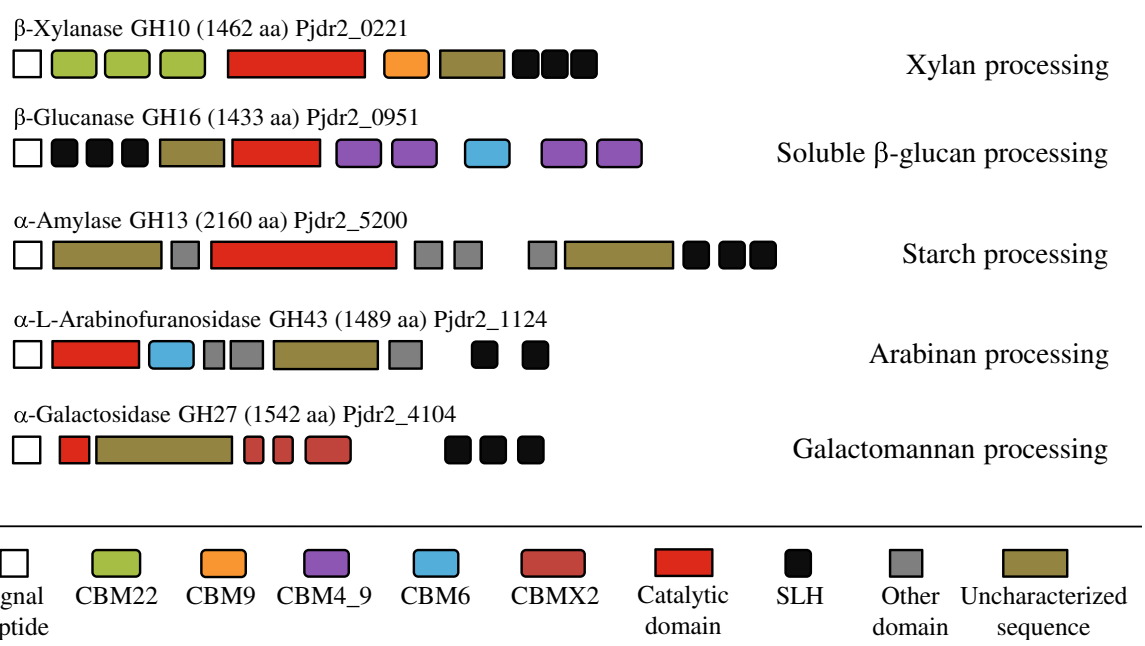

Fig. 4 Multimodular cell-associated enzymes from Pjdr2. A diagram representing the domain architecture of the three surface anchored enzymes central to the utilization of xylan, barley $\beta$-glucan and starch as reported in this work along with two others, further representing the broad use of SLH mediated surface localization of enzymes for polysaccharide assimilation in Pjdr2 as demonstrated in Table 5. Coding sequence locus tag accession numbers are provided as Pjdr2_\#\#\#

generation of oligosaccharides close to the cell surface without diffusion into the medium and hence couples the depolymerization process with assimilation by $\mathrm{ABC}$ transporters for intracellular processing and metabolism. These systems for polysaccharide utilization with minimized secretion of extracellular glycoside hydrolases coupled to transport of oligosaccharides in lieu of simple monomeric sugars potentially affords a significant conservation of cellular energy in the form of ATP as described for the processing of cellulose by C. thermocellum.

Based on increased expression levels of genes during growth on multiple polysaccharides a regulatory connection is observed between utilization of barley $\beta$-glucan, starch and xylans. Barley $\beta$-glucan induces genes involved in extracellular depolymerization and assimilation specific to soluble $\beta-1,3(4)$-glucan. However, it also induces many of the genes shown to play a prominent role in the xylan utilization systems [1]. Although xylans do not induce genes specific to barley $\beta$-glucan utilization, they do induce genes belonging to the starch utilization system. When Pjdr2 was grown on starch, no genes specific to xylan or barley $\beta$-glucan utilization were found to be induced. These studies show the transcriptional induction and repression strategies evolved in Pjdr2 for utilizing a variety of polysaccharides.

Interestingly, induction of the starch utilization genes with growth on xylan results in increased expression of amy $13 A_{1}$ and $a m y 13 A_{3}$ while the $a m y 13 A_{2}$ gene encoding the large surface anchored amylase is expressed just enough to meet the significance selection cutoff (4-fold). This same pattern is also observed with growth on maltose. It appears the elevated expression of the amy $13 A_{2}$ gene is specific for starch and the non-starch substrates which activate the expression of the starch utilization regulon (including $a m y 13 A_{3}$ ) may poise Pjdr2 for rapid response to starch availability.

SLH domains appear to play a vital role in interaction of Pjdr2 with its native environment. The 77 SLH domain-containing proteins encoded in the genome of Pjdr2 highlight the expanded use of this domain for cell wall associations and also hints to a modus operandi, at least regarding an approach to polymeric substrate utilization.

\section{Conclusion}

The genome of Pjdr2 comprises genes encoding extracellular cell-associated depolymerizing enzymes to bioprocess various plant polysaccharides and these include xylans, soluble $\beta$-glucans, starch, and also arabinans and galactomannans (Fig. 4). The polysaccharide utilization systems in Pjdr2 serve as potential candidates for further evaluation or for introduction into other related fermentative bacteria to serve as biocatalysts to achieve direct conversion of non-cellulosic biomass to desired products. Preliminary studies have shown the ability of Pjdr2 to produce fermentative products including lactate, acetate, and ethanol from xylans, $\beta-1,3(4)$-glucans, and starch, under oxygen limiting conditions (unpublished). The potential of Pjdr2 to produce individual cellassociated glycoside hydrolases for processing noncellulosic polysaccharides is an alternative strategy to the cell-associated cellulosome complexes evolved by cellulolytic Clostridium [14]. Pjdr2 may be considered for direct bioprocessing of hemicelluloses or may be co-cultured with cellulolytic organisms tolerant of 
microaerophilic conditions for conversion of biomass to targeted products. Pjdr2 is a candidate for further development as a biocatalyst for consolidated bioprocessing of biomass derived from energy crops and agricultural residues to targeted biofuels and chemicals.

\section{Methods}

\section{Reagents}

The carbohydrates xylose, arabinose, glucose, maltose and cellobiose were of the highest purity available. The starch was purchased from Sigma-Aldrich (St. Louis, MO, USA) and was reported to be pure. Soluble low-viscosity barley $\beta$-glucan (Product No. P-BGBL, Lot 100402a) was purchased from Megazyme International (Wicklow, Ireland) and was reported to contain $<0.1 \%$ arabinoxylan and $<0.31 \%$ starch. The SG and SO xylans were purified from ground sweetgum wood [2, 43] and sorghum stalk bagasse [7] as previously described [44, 45] using standard procedures.

\section{Growth of Pjdr2}

Pjdr2 was routinely cultivated and growth for RNA isolation as described previously [1-4]. A total of 11 growth conditions were considered in this study. These include barley $\beta$-glucan (B) and starch (S) along with their representative dimeric and simple sugars cellobiose $(C)$, maltose $(M)$ and glucose $(G)$, respectively. The sample preparation and RNA-seq data acquisition portion of this manuscript overlaps with a recently published xylan utilization transcriptome [1] which studied the polysaccharide and simple sugar substrates sweetgum wood glucuronoxylan (SG), sorghum stalk glucuronoarabinoxylan (SO), xylose (X) and arabinose (A). Together these paired studies included yeast extract (YE) and sweetgum wood glucuronoxylan without YE supplementation (SGnoYE) as control growth conditions for a total of eleven conditions studied. The characteristics of all growth conditions were defined prior to RNA studies to determine the early mid-exponential phase for harvesting the cells for RNA isolation. Cells were inoculated into $2 \mathrm{ml}$ of $1 \%$ yeast extract (YE) with Zucker-Hankin $(\mathrm{ZH})$ [46] salt medium in $16 \times 100 \mathrm{~mm}$ culture tubes and grown overnight at $30{ }^{\circ} \mathrm{C}$, with an orbital rotation of $250 \mathrm{rpm}$ or with a Roto-torque positioned at a $45^{\circ}$ angle set at high mode and speed 8 . After $24 \mathrm{~h}$ the optical density at $600 \mathrm{~nm}\left(\mathrm{OD}_{600}\right)$ was measured and cells were harvested $(13,000 \mathrm{rpm}, 1 \mathrm{~min})$ to start a sub-culture with $2 \%$ inoculum in $15 \mathrm{ml}$ of $1 \%$ YE in $\mathrm{ZH}$ medium in a $250 \mathrm{ml}$ flask. These cultures were grown at $30{ }^{\circ} \mathrm{C}$ and $250 \mathrm{rpm}$ using a G-2 gyrotary shaker (New Brunswick Scientific) for approximately $6 \mathrm{~h}$ to an $\mathrm{OD}_{600}$ of $0.5-0.8$. The cells were harvested to make an initial inoculation with starting $\mathrm{OD}_{600}$ of 0.04 in $15 \mathrm{ml}$ of desired growth media for this study. These sample cultures were then grown at $30{ }^{\circ} \mathrm{C}$ and $250 \mathrm{rpm}$ until the empirically predetermined early to mid-exponential harvest time for a given condition, generally $\mathrm{OD}_{600} 0.4-0.8$ for carbon supplemented and 0.25 for yeast extract. Growth conditions for RNA sequencing (RNA-seq) transcriptomic analysis consisted of ZH media with $0.5 \%$ carbohydrate and $0.5 \% \mathrm{YE}$, except in two control conditions. The YE control consisted of $0.5 \%$ YE with no carbohydrate and SG control (SGnoYE) with $0.5 \%$ sweetgum $\mathrm{GX}_{\mathrm{n}}$ in $\mathrm{ZH}$ medium without $\mathrm{YE}$. The culture aliquots used for RNA isolation were streaked onto xylan agar plates to confirm the purity of the cultures.

\section{RNA isolation}

For each of the 11 growth conditions, three parallel cultures were grown for a total of 33 RNA isolations. The amount of cells harvested from each culture was determined through empirical analysis of previous growth studies. Total RNA was isolated from early midexponential growing cultures using the RNeasy Protect Bacteria Mini Kit from Qiagen (Valencia, CA, USA) without the use of the RNA Protect reagent. Cells were lysed according to protocol four in the RNAprotect Bacterial Reagent Handbook (2nd edition) with application of lysozyme and Proteinase $\mathrm{K}$ as instructed. RNA was purified from the resulting cell lysate using the RNeasy column (protocol seven of the handbook) with oncolumn RNase-free DNase treatment to remove traces of DNA (Appendix B of the handbook) or in some cases using the TURBO-DNA-free kit from Ambion (Life Technologies, Carlsbad, CA, USA). Total RNA was quantified by absorbance at $260 \mathrm{~nm}$ and the purity was assessed with the $260 / 280 \mathrm{~nm}$ absorbance ratio. Absence of DNA in the RNA preparations was verified by PCR. In some cases the RNeasy on-column DNase treatment was applied and treated again if required.

The RNA preparations were submitted for Bioanalyzer (Agilent, Santa Clara, CA, USA) analysis at the University of Wisconsin Biotechnology Center or The University of Florida Interdisciplinary Center for Biotechnology Research to verify the absence of RNA degradation. The RNA specific quantification of the samples was performed with the Qubit fluorimeter (Life Technologies, Carlsbad, CA) prior to sample submission to the Joint Genome Institute (JGI), Walnut Creek, CA,.

\section{RNA sequencing and data analysis}

RNA sequencing was performed by the Joint Genome Institute (JGI), US Department of Energy, Walnut Creek, CA, as previously described [1]. Briefly, rRNA-depleted RNA was fragmented using divalent cations and high temperature. Fragmented RNA was reverse transcribed using random hexamers and Superscript II (Invitrogen) followed by second strand synthesis. The fragmented cDNA was treated to allow end-pair A-tailing adapter 
ligation and 10 cycles of PCR. Libraries were quantified by qPCR. The libraries were sequenced using the Illumina HiSeq sequencing platform utilizing a TruSeq paired-end cluster kit, v3, and 161 Illumina's cBot instrument to generate clustered flow cells for sequencing. Sequencing of the flow cells was performed on the Illumina HiSeq2000 sequencer using a TruSeq SBS sequencing kit 200 cycles, v3, following a $2 \times 100$ indexed run recipe. Raw sequence read data was filtered using BBDuk (filterk $=27$, trimk $=27$; https: //sourceforge.net $/$ projects/bbmap/) to remove Illumina adapters, known Illumina artifacts, phiX, trim Illumina adapters from the right end of the read and quality-trim the right end of the read to Q6. Resulting reads containing one or more ' $\mathrm{N}$ ', or with quality scores (before trimming) averaging less than 10 over the read, or length under $33 \mathrm{bp}$ after trimming, were discarded.

The filtered raw data files were analyzed using ArrayStar ver. 12.2 software from DNASTAR (Madison, Wisconsin). The results from all growth conditions (in triplicates) were mapped to the annotated genome, averaged and normalized across the entire 11 conditions for comparisons. ANOVA analysis was performed to assess gene data quality with respect to the global dataset. The final output was provided as RPKM values (Reads Per Kilobase per Million reads sequenced). In ArrayStar, statistical analysis for comparisons of two conditions was performed with the moderated $t$-test and adjusted $p$-values were calculated using the FDR (Benjamini Hochberg) method [47]. Unless otherwise stated, the expression of genes discussed in this study is based upon a fold difference relative to YE control. Data with $p$-values less than 0.05 were considered to be significant.

\section{Gene annotation and analysis}

Functional roles were assigned to genes based on analysis by BLASTp from NCBI (http://www.ncbi.nlm.nih.gov/) [48, 49], IMG database (http://img.jgi.doe.gov/) or Pfam (http://pfam.xfam.org/) [50]. For genome analysis of the SLH containing genes, the first and third SLH domains of xylanase XynA1 were blasted against the genome and the two resulting datasets combined and made nonredundant. Operon predictions were based on in silico analysis using the PePPER webserver (http://server.molgenrug.nl/) [51]. The genes from the genome of Pjdr2 are identified by their locus tags. The locus tags are identified as Pjd2_\#\#\#, where \#\#\#\# represents the 4-digit gene number used for gene identification in this study. The filtered raw data was processed using ArrayStar. This processed data is available in the supplemental material (Additional file 2). The data represented in the Tables have expression data rounded off to 1 or 2 decimal points, and the $p$-values converted to 2 decimal points. Raw data is available as described in Availability of Supporting Data section.

\section{Availability of supporting data}

The data sets supporting the results of this article are available in the JGI Genome Portal repository, Project ID 1023680 located at http://genome.jgi.doe.gov/pages/ projectStatus.jsf?db=Paespnscriptome.

\section{Additional files}

Additional file 1: A table summarizing the proteins belonging to
the xylan utilization systems in Pjdr2 as previously determined [1].
(DOCX $19 \mathrm{~kb}$ )
Additional file 2: Total transcriptome data representing all growth
conditions from this study. The data table consists of Pjdr2 gene locus
tag ID (accession numbers), in silico annotation results, per transcript
ANOVA analysis results, calculated transcript quantity given as RPKM
values (Reads Per Kilobase per Million reads sequenced), fold change
data for each gene in every growth condition relative to the YE control
with corresponding $p$-values (moderated t-test), fold change data for
each gene in the glucan containing growth conditions relative to their
constituent sugars with corresponding $p$-values and fold change data
for each gene in the xylan growth conditions relative to xylose and
arabinose with corresponding $p$-values. Analyses were performed using
DNASTAR Arraystar $v 12$ (Madison, WI). (XLSX 6349 kb)

Competing interests

The authors declare that they have no competing interests.

\section{Authors' contributions}

NS, CC, VC, JFP and FJSJ designed the research plan. NS and FJSJ performed microbiological studies, prepared RNA, performed RNA quality control, extracted and tabulated RNA-seq data and prepared the draft manuscript. CC contributed to RNA quality assessment, performed RNA-seq data assembly and normalization and contributed to data mining. NS, CC, VC, JFP and FJSJ contributed to editing the manuscript and figures. All authors have read and approved the final version of the manuscript.

\section{Acknowledgements}

We appreciate the assistance provided by Dr. L. O. Ingram, Dr. K. T. Shanmugam, and John D. Rice all from the Department of Microbiology and Cell Science, University of Florida, Gainesville, FL, for useful advice. We also thank Dr. Daniel Cullen for his expertise and helpful discussions regarding transcriptome studies and Diane Dietrich for professional support, both from the USDA, US Forest Service, Forest Products Laboratory, Madison, WI. We would like to thank Christa Pennacchio for managing the RNA sequencing project and also the other members of the Joint Genome Institute, Walnut Creek, CA, for conducting the RNA sequencing work which was supported by the Office of Science of the US Department of Energy under Contract No. DE-AC02-05CH11231. This research was supported by Biomass Research \& Development Initiative Competitive Grant No. 2011-10006-30358 from the USDA National Institute of Food and Agriculture, and by Florida Energy Systems Consortium, State University System of FL, Project No. 00077818.

Received: 22 October 2015 Accepted: 5 February 2016

Published online: 24 February 2016

\section{References}

1. Sawhney N, Crooks C, St John FJ, Preston JF. Transcriptomic analysis of xylan utilization systems in Paenibacillus sp. JDR-2. Appl Environ Microbiol. 2015:81(4):1490-501.

2. St John FJ, Rice JD, Preston JF. Paenibacillus sp. strain JDR-2 and XynA1: a novel system for methylglucuronoxylan utilization. Appl Environ Microbiol. 2006;72(2):1496-506.

3. Nong G, Rice JD, Chow V, Preston JF. Aldouronate utilization in Paenibacillus sp. strain JDR-2: Physiological and enzymatic evidence for coupling of extracellular depolymerization and intracellular metabolism. Appl Environ Microbiol. 2009;75(13):4410-8. 
4. Chow V, Nong G, Preston JF. Structure, function, and regulation of the aldouronate utilization gene cluster from Paenibacillus sp. strain JDR-2. J Bacteriol. 2007;189(24):8863-70.

5. Chow V, Nong G, St John FJ, Rice JD, Dickstein E, Chertkov O, et al. Complete genome sequence of Paenibacillus sp. strain JDR-2. Stand Genomic Sci. 2012:6(1):1-10.

6. Lombard V, Ramulu HG, Drula E, Coutinho PM, Henrissat B. The carbohydrate-active enzymes database (CAZy) in 2013. Nucleic Acids Res. 2014;42(D1):D490-5

7. Sawhney N, Preston JF. GH51 Arabinofuranosidase and Its role in the Methylglucuronoarabinoxylan utilization system in Paenibacillus sp. Strain JDR-2. Appl Environ Microbiol. 2014;80(19):6114-25.

8. Shulami S, Gat O, Sonenshein AL, Shoham Y. The glucuronic acid utilization gene cluster from Bacillus stearothermophilus T-6. J Bacteriol. 1999;181(12):3695-704.

9. Chhabra SR, Shockley KR, Conners SB, Scott K, Wolfinger RD, Kelly RM. Carbohydrate-induced differential gene expression patterns in the hyperthermophilic bacterium Thermotoga maritima. J Biol Chem. 2003;278:7540-52.

10. Izquierdo JA, Pattathil S, Guseva A, Hahn MG, Lynd LR. Comparative analysis of the ability of Clostridium clariflavum strains and Clostridium thermocellum to utilize hemicellulose and unpretreated plant material. Biotechnol Biofuels. 2014;7(1):136

11. Shulami S, Shenker O, Langut Y, Lavid N, Gat O, Zaide G, et al. Multiple regulatory mechanisms control the expression of the Geobacillus stearothermophilus gene for extracellular xylanase. J Biol Chem. 2014;289(37):25957-75.

12. Preston JF, Hurlbert JC, Rice JD, Ragunathan A, St John FJ. Microbial strategies for the depolymerization of glucuronoxylan: leads to biotechnological applications of endoxylanases. In: Applications of Enzymes to Lignocellulosics. Washington D.C: American Chemical Society; 2003. p. 191-210.

13. Gold ND, Martin VJJ. Global view of the Clostridium thermocellum cellulosome revealed by quantitative proteomic analysis. J Bacteriol. 2007;189(19):6787-95.

14. Smith SP, Bayer EA. Insights into cellulosome assembly and dynamics: from dissection to reconstruction of the supramolecular enzyme complex. Curr Opin Struct Biol. 2013;23(5):686-94.

15. Doi RH, Kosugi A. Cellulosomes: plant-cell-wall-degrading enzyme complexes. Nat Rev Microbiol. 2004;2(7):541-51.

16. Lynd LR, Weimer PJ, Van ZyI WH, Pretorius IS. Microbial cellulose utilization: fundamentals and biotechnology. Microbiol Mol Biol Rev. 2002;66(3):506-77.

17. Pan L. Starch utilization in Paenibacillus sp. strain JDR-2: Depolymerization catalyzed by cell associated multi-modular alpha-amylase Masters Thesis. Gainesville, Florida: University of Florida; 2014.

18. Chow V, Kim Y, Rhee M, Sawhney N, St John F, Nong G et al. Definition of a 1,3-1,4- $\beta$-glucan-utilization regulon in Paenibacillus sp. JDR-2. Appl Environ Microbiol. 2016; doi:10.1128/AEM.03526-15. In-Press Epub.

19. Kosugi A, Murashima K, Tamaru Y, Doi RH. Cell surface anchoring role of $\mathrm{N}$-terminal surface layer homology domains of Clostridium cellulovorans EngE. J Bacteriol. 2002;184(4):884-8.

20. Ali MK, Fukumura M, Sakano K, Karita S, Kimura T, Sakka K, et al. Cloning, sequencing, and expression of the gene encoding the Clostridium stercorarium xylanase C in Escherichia coli. Biosci Biotechnol Biochem. 1999;63(9):1596-604.

21. Feng J-X, Karita S, Fujino E, Fujino T, Kimura T, Sakka K, et al. Cloning, sequencing, and expression of the gene encoding a cell-bound multi-domain xylanase from Clostridium josui, and characterization of the translated product. Biosci Biotechnol Biochem. 2000;64(12):2614-24.

22. Ozdemir I, Blumer-Schuette SE, Kelly RM. S-layer homology domain proteins Csac_0678 and Csac_2722 are implicated in plant polysaccharide deconstruction by the extremely thermophilic bacterium Caldicellulosiruptor saccharolyticus. Appl Environ Microbiol. 2012;78(3):768-77.

23. Liu S-Y, Gherardini FC, Matuschek M, Bahl H, Wiegel J. Cloning, sequencing, and expression of the gene encoding a large S-layer-associated endoxylanase from Thermoanaerobacterium sp. strain JW/SL-YS 485 in Escherichia coli. J Bacteriol. 1996;178(6):1539-47.

24. Lazaridou A, Biliaderis C. Molecular aspects of cereal $\beta$-glucan functionality: Physical properties, technological applications and physiological effects. J Cereal Sci. 2007;46(2):101-18.

25. Jeng $W-Y$, Wang N-C, Lin C-T, Shyur L-F, Wang AH-J. Crystal structures of the laminarinase catalytic domain from Thermotoga maritima MSB8 in complex with inhibitors essential residues for $\beta-1$, 3-and $\beta$-1, 4-glucan selection. J Biol Chem. 2011;286(52):45030-40.
26. Wolf M, Geczi A, Simon O, Borriss R. Genes encoding xylan and $\beta$-glucan hydrolysing enzymes in Bacillus subtilis: characterization, mapping and construction of strains deficient in lichenase, cellulase and xylanase. Microbiology. 1995;141(2):281-90.

27. David MH, Günther H, Röper H. Catalytic properties of Bacillus megaterium amylase. Starch-Starke. 1987:39(12):436-40.

28. Metz RJ, Allen LN, Cao TM, Zeman NW. Nucleotide sequence of an amylase gene from Bacillus megaterium. Nucleic Acids Res. 1988;16(11):5203.

29. Mathupala S, Saha BC, Zeikus JG. Substrate competition and specificity at the active site of amylopullulanase from Clostridium thermohydrosulfuricum. Biochem Biophys Res Commun. 1990;166(1):126-32.

30. Cho H-Y, Kim Y-W, Kim T-J, Lee H-S, Kim D-Y, Kim J-W, et al. Molecular characterization of a dimeric intracellular maltogenic amylase of Bacillus subtilis SUH4-2. Biochim Biophys Acta. 2000;1478(2):333-40.

31. Schönert S, Seitz S, Krafft H, Feuerbaum E-A, Andernach I, Witz G, et al. Maltose and maltodextrin utilization by Bacillus subtilis. J Bacteriol. 2006:188(11):3911-22.

32. Mizanur RM, Griffin AK, Pohl NL. Recombinant production and biochemical characterization of a hyperthermostable a-glucan/maltodextrin phosphorylase from Pyrococcus furiosus. Archaea. 2008;2(3):169-76.

33. Xavier KB, Peist R, Kossmann M, Boos W, Santos H. Maltose metabolism in the hyperthermophilic archaeon Thermococcus litoralis: purification and characterization of key enzymes. J Bacteriol. 1999;181(11):3358-67.

34. Ampomah OY, Avetisyan A, Hansen E, Svenson J, Huser T, Jensen JB, et al. The thuEFGKAB operon of rhizobia and Agrobacterium tumefaciens codes for transport of trehalose, maltitol, and isomers of sucrose and their assimilation through the formation of their 3-keto derivatives. J Bacteriol. 2013;195(17):3797-807.

35. Shulami S, Raz-Pasteur A, Tabachnikov O, Gilead-Gropper S, Shner I, Shoham $Y$. The L-arabinan utilization system of Geobacillus stearothermophilus. J Bacteriol. 2011;193(11):2838-50.

36. Park Y-C, Jun SY, Seo J-H. Construction and characterization of recombinant Bacillus subtilis JY123 able to transport xylose efficiently. J Biotechnol. 2012;161(4):402-6.

37. Koehler P, Wieser H. Chemistry of Cereal Grains. In: Gobbetti M, Gänzle M, editors. Handbook on sourdough biotechnology. New York: Springer; 2013. p. 11-45.

38. Fukuda $M$, Watanabe $S$, Yoshida $S$, Itoh $H$, Itoh $Y$, Kamio $Y$, et al. Cell surface xylanases of the glycoside hydrolase family 10 are essential for xylan utilization by Paenibacillus sp. W-61 as generators of xylo-oligosaccharide inducers for the xylanase genes. J Bacteriol. 2010;192(8):2210-9.

39. Kern J, Wilton R, Zhang R, Binkowski TA, Joachimiak A, Schneewind O. Structure of surface layer homology (SLH) domains from Bacillus anthracis surface array protein. J Biol Chem. 2011;286(29):26042-9.

40. Boraston A, Bolam D, Gilbert H, Davies G. Carbohydrate-binding modules: fine-tuning polysaccharide recognition. Biochem J. 2004;382:769-81.

41. Janesch B, Messner P, Schäffer C. Are the surface layer homology domains essential for cell surface display and glycosylation of the S-layer protein from Paenibacillus alvei CCM 2051 T? J Bacteriol. 2013;195(3):565-75.

42. Zhang Y-HP, Lynd LR. Cellulose utilization by Clostridium thermocellum: bioenergetics and hydrolysis product assimilation. Proc Natl Acad Sci U S A. 2005;102(20):7321-5.

43. Hurlbert JC, Preston JF. Functional characterization of a novel xylanase from a corn strain of Erwinia chrysanthemi. J Bacteriol. 2001;183(6):2093-100.

44. Jones J, Purves C, Timell T. Constitution of a 4-O-methylglucuronoxylan from the wood of trembling aspen (Populus tremuloides Michx.). Can J Chem. 1961;39(5):1059-66.

45. Kardošová A, Ebringerová A, Alföldi J, Nosál'ová G, Matáková T. Hřibalová V. Structural features and biological activity of an acidic polysaccharide complex from Mahonia aquifolium (Pursh) Nutt. Carbohydr Polym. 2004;57(2):165-76.

46. Zucker M, Hankin L. Regulation of pectate lyase synthesis in Pseudomonas fluorescens and Erwinia carotovora. J Bacteriol. 1970;104(1):13-8.

47. Benjamini $Y$, Hochberg Y. Controlling the false discovery rate: a practical and powerful approach to multiple testing. J Roy Stat Soc Ser B (Stat Method). 1995;57(1):289-300

48. Johnson M, Zaretskaya I, Raytselis Y, Merezhuk Y, McGinnis S, Madden TL. NCBI BLAST: a better web interface. Nucleic Acids Res. 2008;36(Web Server issue):W5-9.

49. Marchler-Bauer A, Anderson JB, Derbyshire MK, DeWeese-Scott C, Gonzales NR, Gwadz M, et al. CDD: a conserved domain database for interactive domain family analysis. Nucleic Acids Res. 2007;35(Database issue):D237-40. 
50. Bateman A, Coin L, Durbin R, Finn RD, Hollich V, Griffiths-Jones S, et al. The Pfam protein families database. Nucleic Acids Res. 2004;32 suppl 1:D138-41.

51. de Jong A, Pietersma H, Cordes M, Kuipers OP, Kok J. PePPER: a webserver for prediction of prokaryote promoter elements and regulons. BMC Genomics. 2012;13(1):299.

Submit your next manuscript to BioMed Central and we will help you at every step:

- We accept pre-submission inquiries

- Our selector tool helps you to find the most relevant journal

- We provide round the clock customer support

- Convenient online submission

- Thorough peer review

- Inclusion in PubMed and all major indexing services

- Maximum visibility for your research

Submit your manuscript at www.biomedcentral.com/submit 